${ }^{1}$ Division of Cardiology, Department of Medicine, Karolinska Institutet, Stockholm, Sweden.

${ }^{2}$ Heart and Vascular Theme, Karolinska University Hospital, Stockholm, Sweden. ${ }^{3}$ Cardiothoracovascular Department, Azienda Sanitaria Universitaria Giuliano Isontina (ASUGI) and University Hospital of Trieste, Trieste, Italy.

${ }^{4}$ These authors contributed equally: Gianluigi Savarese Davide Stolfo.

凶e-mail:

lars.lund@alumni.duke.edu

https://doi.org/10.1038/

s41569-021-00605-5

\title{
Heart failure with mid-range or mildly reduced ejection fraction
}

Gianluigi Savarese ${ }^{1,2,4}$, Davide Stolfo ${ }^{1,3,4}$, Gianfranco Sinagra (i) $^{3}$ and Lars H. Lund (iD) ${ }^{1,2 凶}$

Abstract | Left ventricular ejection fraction (EF) remains the major parameter for diagnosis, phenotyping, prognosis and treatment decisions in heart failure. The 2016 ESC heart failure guidelines introduced a third EF category for an EF of 40-49\%, defined as heart failure with mid-range EF (HFmrEF). This category has been largely unexplored compared with heart failure with reduced $\mathrm{EF}$ ( $\mathrm{HFrEF}$; defined as $\mathrm{EF}<40 \%$ in this Review) and heart failure with preserved $\mathrm{EF}$ (HFpEF; defined as $\mathrm{EF} \geq 50 \%$ ). The prevalence of $\mathrm{HFmrEF}$ within the overall population of patients with $\mathrm{HF}$ is $10-25 \%$. HFmrEF seems to be an intermediate clinical entity between HFrEF and $\mathrm{HFpEF}$ in some respects, but more similar to HFrEF in others, in particular with regard to the high prevalence of ischaemic heart disease in these patients. $\mathrm{HFmrEF}$ is milder than $\mathrm{HFrEF}$, and the risk of cardiovascular events is lower in patients with HFmrEF or HFpEF than in those with HFrEF. By contrast, the risk of non-cardiovascular adverse events is similar or greater in patients with HFmrEF or HFpEF than in those with HFrEF. Evidence from post hoc and subgroup analyses of randomized clinical trials and a trial of an SGLT1-SGLT2 inhibitor suggests that drugs that are effective in patients with HFrEF might also be effective in patients with HFmrEF. Although the $\mathrm{EF}$ is a continuous measure with considerable variability, in this comprehensive Review we suggest that HFmrEF is a useful categorization of patients with HF and shares the most important clinical features with $\mathrm{HFrEF}$, which supports the renaming of $\mathrm{HFmrEF}$ to $\mathrm{HF}$ with mildly reduced $\mathrm{EF}$.

Heart failure (HF) is a global pandemic with an increasing prevalence. Drivers of the growing prevalence of $\mathrm{HF}$ are ageing of the population, improved survival after myocardial infarction, and improved treatment and survival of patients with $\mathrm{HF}^{1-4}$. As a result, the burden of HF-related hospitalizations and costs are increasing, with the total costs for HF in 2012 estimated to be US $\$ 30.7$ billion, of which more than two-thirds is attributable to direct medical costs, and projections suggesting an increase of $127 \%$ by 2030 (REF. ${ }^{7}$ ). Despite the availability of effective therapies, the prognosis of patients with HF remains poor ${ }^{8}$. HF is the leading cause of hospitalization among adults, and 1-year mortality is $10-35 \%$ in various population-wide registries, and is much higher in patients with advanced $\mathrm{HF}^{9-12}$.

Left ventricular ejection fraction (EF), generally measured by echocardiography, remains the cornerstone of HF diagnosis, characterization, prognosis, patient triage and treatment selection. The clinical use of EF has flaws, which are described below in detail. Advanced multivariable analytics (such as machine learning and other methods for patient clustering and phenotyping) as well as other parameters have demonstrated a better calibration and discrimination for survival than the use of EF alone ${ }^{13}$. Nevertheless, EF remains the primary parameter for HF characterization and the primary inclusion criterion for clinical trials of HF. Until better measurements than the EF are available that meet all the needs for HF characterization, the data described in this Review might be relevant for both clinicians and clinical trialists ${ }^{6,14-16}$.

$\mathrm{HF}$ with reduced $\mathrm{EF}$ (HFrEF; defined as $\mathrm{EF}<40 \%$ ) is well characterized, and effective therapies for patients with HFrEF are available. The term HF with preserved $\mathrm{EF}$ (HFpEF) has long been used to describe patients with HF signs and symptoms and an EF that was variably defined as $>40 \%,>45 \%$ or $\geq 45 \%$, or $>50 \%$ or $\geq 50 \%$. For these patients, no clinical trial to date has demonstrated clear benefits of therapy ${ }^{17}$. In the 2016 ESC HF guidelines, a separate entity, HF with mid-range EF (HFmrEF; defined as EF 40-49\%), was introduced to foster research in this EF range, which has been less investigated than HFrEF $(\mathrm{EF}<40 \%)$ and HFpEF $(\mathrm{EF} \geq 50 \%)^{6}$. Extensive subsequent research confirms that HFmrEF has some intermediate features between HFrEF and HFpEF but also suggests distinct similarities between HFmrEF and HFrEF that warrant the term HF with 'mildly reduced' $\mathrm{EF}$, as has also been proposed by other authors in the past 2 years ${ }^{18-20}$. In this Review, we provide a comprehensive overview of the epidemiology, clinical profile, 


\section{Key points}

- Heart failure (HF) with mildly reduced ejection fraction (EF) (HFmrEF) has been extensively studied, generally using an EF of $40-49 \%$, and accounts for up to $25 \%$ of patients with HF.

- On the basis of contemporary trials and definitions, HFmrEF might be defined as an EF of $41-49 \%$.

- HFmrEF is an intermediate HF type between HF with preserved EF (HFpEF) and HF with reduced $\mathrm{EF}$ (HFrEF) for some characteristics but is more similar to HFrEF for others, especially for the high prevalence of ischaemic heart disease.

- HFmrEF and HFpEF are milder forms of HF than HFrEF and are associated with a lower risk of cardiovascular and $\mathrm{HF}$ events but with a similar or greater risk of non-cardiovascular adverse events.

- Clinical trials on therapies for HFpEF enrolled patients with an ejection fraction of $>40 \%$ or $\geq 45 \%$ and did not demonstrate a clear treatment effect; however, subgroup and post hoc analyses suggest that some therapies for HFrEF might also be effective in HFmrEF.

- These arguments support the current redefinition of HFmrEF as HF with mildly reduced $\mathrm{EF}$ instead of $\mathrm{HF}$ with mid-range $\mathrm{EF}$.

prognosis and potential treatment of HFmrEF, which we define as HF with mildly reduced EF.

\section{EF in heart failure}

$\mathrm{EF}$ is a normally distributed, continuous measure in the general population ${ }^{21}$, but has a bimodal distribution among patients with incident $\mathrm{HF}^{22,23}$, which supports the current concept that HF has distinct forms. According to the definition in European and US guidelines, the normal EF range is $52-72 \%$ in men and $54-74 \%$ in women, with normal values plus or minus standard deviation being $62 \pm 5 \%$ for men and $64 \pm 5 \%$ for women ${ }^{24,25}$. Given that HFmrEF is defined by a tighter EF range than HFrEF and HFpEF, measurement variability in

\section{Box 1 | Implications of variability in EF measurements}

- Intraobserver and interobserver variability of standard echocardiographic left ventricular ejection fraction (EF) assessment is reported to be $8-21 \%$ and $6-13 \%$, respectively ${ }^{173}$

- Sources of measurement variability include

- Digit-rounding bias: the tendency is to report EF with numbers ending in 0 or 5 , for example as $40 \%$ instead of $39 \%$ or $41 \%$. Therefore, whether heart failure with mildly reduced $\mathrm{EF}(\mathrm{HFmrEF})$ is defined as $\mathrm{EF} 40-49 \%$ or $41-49 \%$ can have major implications for estimating the prevalence of $\mathrm{HFmrEF}$

- Regression to the meanVisual estimation: although reliability has been reported as sufficient $^{174,175}$, visual estimation can underestimate true EF values

- Poor image quality

- Measurement error, in particular in the setting of rapid and/or irregular ventricular rhythm

- Consequences of measurement variability

- Misclassification: in randomized clinical trials, estimation of EF at local sites can lead to the misclassification of patients for enrolment. In the TOPCAT trial ${ }^{176}$, core laboratory measurements would have reclassified about $20 \%$ of the $E F$ measurements

- Future perspectives

- Optimization of automated algorithms for the analysis of EF

- Systematic adoption of core laboratories fulfilling standard requirements for randomized clinical trials that are based on $\mathrm{EF}^{66}$

- Improved agreement between imaging techniques: substantial variability in the estimation of EF has been reported across imaging modalities even when quantified by core laboratories ${ }^{177}$

- Integrating EF with additional criteria (such as biomarkers and multivariable scores and algorithms) for more accurate and precise categorization of heart failure
EF quantification can have important implications for $\operatorname{HFmrEF}^{26}$ (BOX 1).

The EF became the predominant tool to characterize, risk stratify and select patients with HF in clinical trials in the 1980s as a result of the design of these trials to select for enrolment only of patients with reduced $\mathrm{EF}$ as an enrichment strategy. Although the concept of HF with 'normal' or 'intact' EF was introduced in the $1980 \mathrm{~s}^{27,28}$, these patients were included in interventional trials only much later.

Various cut-off values for EF have been proposed and used to differentiate reduced from preserved EF for trial design purposes, with most trials of HFrEF considering an EF of $<45 \%{ }^{29},<40 \%$ or $\leq 40 \%{ }^{30-36}$ but also $\leq 35 \%^{37-42}, \leq 30 \%{ }^{43,44}$ or even $<25 \%{ }^{45}$, to target patients with more severe HF and/or as an enrichment strategy (for example, to increase the rates of cardiovascular events and therefore reduce the sample size needed to observe an effect of the tested treatment if such an effect is present $)^{46}$. The rationale for the clinical use of EF for HF classification is supported by evidence from large, randomized clinical trials (RCTs), and ensuing guidelines and regulatory approvals, showing that drugs and devices for the treatment of HF improve outcomes in patients with reduced $\mathrm{EF}(\mathrm{EF} \leq 40 \%)$, whereas no equivalent evidence is available in patients with higher $\mathrm{EF}^{6}$. Therefore, the EF identifies two different HF phenotypes according to treatment response. Additionally, the choice of using the cut-off value of $\mathrm{EF}=40 \%$ to define $\mathrm{HFrEF}$ is further supported by the higher risk of adverse cardiovascular outcomes in patients with $\mathrm{EF}<40 \%$ than in patients with $\mathrm{EF} \geq 40 \%$ observed in several studies ${ }^{47-50}$. The definition of preserved EF has varied across different trials, with HFpEF defined as EF $>40 \%$ or $\geq 40 \%$ in the CHARM-Preserved ${ }^{51}$, EMPEROR-Preserved ${ }^{52}$, DELIVER $^{53}$, SPIRIT-HF ${ }^{54}$ and SPIRRIT-HFpEF ${ }^{55}$ trials $^{56}$, as EF $\geq 45 \%$ in the TOPCAT ${ }^{57}$, I-PRESERVE ${ }^{58}$ and PARAGON-HF ${ }^{59}$ trials, and as $\mathrm{EF} \geq 50 \%$ in the 2020 SOLOIST-WHF trial ${ }^{60}$. The inconsistent inclusion in trials of HFpEF of patients with EF of 40-49\%, together with the above reported definition of normal EF, led to questions over how to characterize patients with $\mathrm{EF}$ $40-49 \%^{24}$. Therefore, in 2016 the ESC guidelines on $\mathrm{HF}$ introduced the term 'mid-range' EF and acknowledged a new HF phenotype between HFpEF and HFrEF, which was named HF with mid-range $\mathrm{EF}$ (HFmrEF) and defined as an EF of $40-49 \%$ in combination with the presence of signs and symptoms of HF, elevated levels of natriuretic peptides in the plasma and evidence of structural heart disease (left ventricular hypertrophy or left atrial remodelling) or the presence of diastolic dysfunction, to ensure a diagnosis of HF in a setting in which the EF is not markedly reduced ${ }^{6}$. However, given that the EF in HFmrEF is below the normal range, why HFmrEF should require more supportive criteria than HFrEF is not readily apparent. The aim of the guidelines committee was to stimulate research into the "underlying characteristics, pathophysiology and treatment of this group of patients" . Analogously, the 2013 ACCF/AHA guidelines on HF defined an EF of 41-49\% as $\mathrm{HF}$ with borderline $\mathrm{EF}$ to differentiate this entity from HFrEF but not include HFpEF ${ }^{61}$. Finally, in the universal 


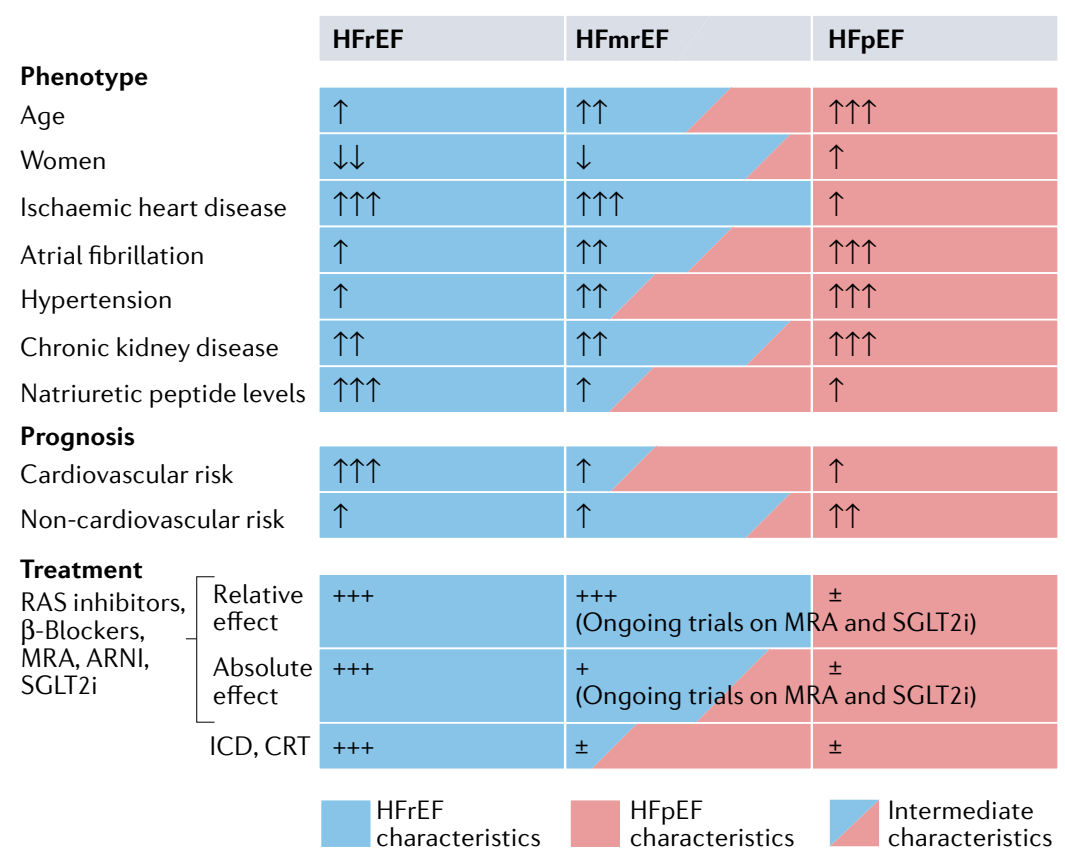

Fig. 1 | Phenotype, risk of cause-specific outcomes and effect of therapies in HFrEF, HFmrEF and HFpEF. Phenotype, risk of cause-specific outcomes and demonstrated or potential effect of treatments across the left ventricular ejection fraction categories of heart failure. Heart failure with mildly reduced ejection fraction (HFmrEF) shares features with both heart failure with reduced ejection fraction (HFrEF), such as a higher prevalence of ischaemic heart disease and less frequent renal impairment, and heart failure with preserved ejection fraction (HFpEF), such as hypertension, milder heart failure symptoms and lower levels of natriuretic peptides. HFmrEF is intermediate between the two categories for age and prevalence of atrial fibrillation. Cardiovascular mortality is lower in patients with $\mathrm{HFmrEF}$ and those with HFpEF than in patients with HFrEF. Non-cardiovascular mortality is lower in patients with $\mathrm{HFmrEF}$ and those with $\mathrm{HFrEF}$ than in patients with $\mathrm{HFpEF}$. Post hoc and subgroup analyses of trials of heart failure suggest a potential benefit of therapy with a mineralocorticoid-receptor antagonist (MRA), angiotensin-receptor blocker-neprilysin inhibitor (ARNI) or sodium-glucose cotransporter 2 inhibitor (SGLT2i) in patients with HFmrEF. $\uparrow$ and $\downarrow$ denote higher or more common and lower or less common, respectively, than in an age-matched control population, with the exception of age, in which $\uparrow$ denotes higher than average among adults; + denotes strength of benefit; \pm denotes insufficient evidence. CRT, cardiac resynchronization therapy; ICD, implantable cardioverter-defibrillator; RAS, renin-angiotensin system.

definition and classification of HF published in 2021, $\mathrm{HFrEF}$ was defined as $\mathrm{EF} \leq 40 \%$, HFpEF as $\mathrm{EF} \geq 50 \%$ and HFmrEF, renamed as HF with mildly reduced EF, as EF $41-49 \%{ }^{19}$. The trials of HFrEF conducted in the past 5 years included patients with $\mathrm{EF} \leq 40 \%$, and given the $5 \%$ interval digit preference, we agree that HFrEF is most reasonably defined as $\leq 40 \%$. However, the 2016 ESC HF guidelines put an EF of $40 \%$ in the HFmrEF category and, therefore, most research since then, including most of the studies discussed in this Review, have considered an EF of $40 \%$ to be part of the HFmrEF range of $\mathrm{EF}^{6}$.

\section{Epidemiology}

The incidence of HF in Western countries is 1-9 cases per 1,000 person-years, with a prevalence of $\sim 2 \%{ }^{62,63}$. The prevalence of HFmrEF within the overall population of patients with $\mathrm{HF}$ is $10-25 \%{ }^{48,64-70}$. However, $\mathrm{HFpEF}$ might be underdiagnosed because the $\mathrm{EF}$ is normal and might be missed in routine clinical care; therefore, the prevalence of HFmrEF might be proportionally lower than that of HFpEF. However, HFmrEF might also be underdiagnosed, considering the error and variability in EF measurement and the presence of milder symptoms in these patients compared with patients with HFrEF, leading clinicians to miss the HF diagnosis in patients with mild or diffuse symptoms and a low-normal or minimally reduced $\mathrm{EF}^{48,64,65,67-71}$. However, if $\mathrm{HFmrEF}$ was defined as an EF of $41-49 \%$ instead of $40-49 \%$, the number of patients diagnosed with HFmrEF would be smaller. In a large, community-based, longitudinal cohort of individuals free from HF at baseline and followed up for $>10$ years, the incidence of HFmrEF was only 6.7 cases per 10,000 person-years versus an incidence of 26.9 and 34.9 cases per 10,000 person-years for HFpEF and HFrEF, respectively, with predictors of incident $\mathrm{HF}$ being consistent across the EF spectrum ${ }^{64}$. In the ESC-HF-LT registry, $24 \%$ of the enrolled patients had HFmrEF, whereas the proportion of patients with HFmrEF in the Swedish SwedeHF registry was $21 \%{ }^{48,65}$. A slightly lower prevalence of HFmrEF was reported in Asian $^{68,70}$, New Zeland ${ }^{68}$ and North American ${ }^{67}$ registries. Finally, in the CHARM programme ${ }^{72}$, which enrolled patients with $\mathrm{HF}$ regardless of EF, $17 \%$ of the patients had HFmrEF

\section{Clinical characteristics of HFmrEF}

Proper characterization and phenotyping of HFmrEF is important because it might support treatment recommendations based on available data and inform the design of future interventional trials in HFmrEF by facilitating appropriate trial selection criteria. HFmrEF is often defined as 'intermediate' (favouring the use of the term 'mid-range') because this classification makes intuitive sense, or as a milder form of HFrEF (favouring the use of the term 'mildly reduced') because of some distinct clinical and treatment response similarities to $\mathrm{HFrEF}^{73}$. Both of these approaches are overly simplistic and ignore the extensive emerging research that characterizes HFmrEF in detail in relation to HFrEF and HFpEF. FIGURE 1 provides a conceptual representation of HFmrEF in relation to HFrEF and HFpEF, and FICS 2,3 provide detailed characteristics from specific data sets.

In US cohorts of patients with HF, patients with an EF of $40-50 \%$ had similar characteristics to patients with HFpEF in terms of higher age, BMI and prevalence of hypertension and atrial fibrillation compared with patients with $\mathrm{HFrEF}^{74-76}$. However, this patient group was more similar to the HFrEF group in terms of sex distribution (more likely to be men) and a higher prevalence of ischaemic heart disease (IHD) than the HFpEF group ${ }^{74-76}$. In the ESC-HF-LT registry, the HFmrEF group shared several characteristics with the HFrEF group, including younger age, male sex, ischaemic aetiology and lower prevalence of atrial fibrillation compared with the HFpEF group ${ }^{65}$. Notably, patients with HFmrEF were less symptomatic (lower NYHA class), less likely to receive diuretics and with overall fewer comorbidities than either patients with HFpEF or patients with HFrEF. A potential explanation for these findings might be that HFmrEF represented a mild form of HFrEF or, in some patients, 


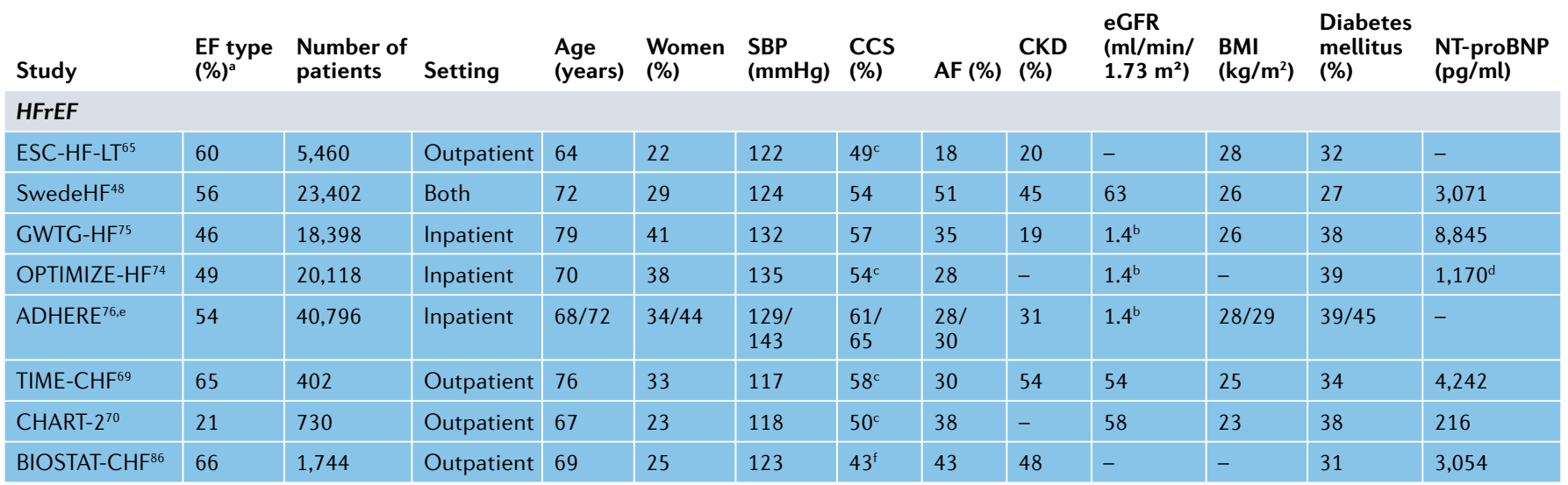

\begin{tabular}{|c|c|c|c|c|c|c|c|c|c|c|c|c|c|}
\hline \multicolumn{14}{|l|}{ HFmrEF } \\
\hline ESC-HF-LT ${ }^{65}$ & 24 & 2,212 & Outpatient & 64 & 32 & 127 & $42^{c}$ & 22 & 17 & - & 29 & 31 & - \\
\hline SwedeHF ${ }^{48}$ & 21 & 9,019 & Both & 74 & 39 & 131 & 53 & 58 & 48 & 62 & 27 & 27 & 2,160 \\
\hline OPTIMIZE-HF 74 & 20 & 7,321 & Inpatient & 74 & 52 & 147 & $49^{c}$ & 33 & - & $1.3^{b}$ & - & 44 & $757^{d}$ \\
\hline $\mathrm{ADHERE}^{76, \mathrm{e}}$ & 23 & 17,045 & Inpatient & 74 & 54 & 150 & 60 & 33 & 31 & $1.3^{\mathrm{b}}$ & 30 & 48 & - \\
\hline BIOSTAT-CHF ${ }^{86}$ & 18 & 416 & Outpatient & 75 & 34 & 129 & $48^{f}$ & 49 & 53 & - & - & 35 & 1,839 \\
\hline
\end{tabular}

\begin{tabular}{|c|c|c|c|c|c|c|c|c|c|c|c|c|c|}
\hline HFpEF & & & & & & & & & & & & & \\
\hline ESC-HF-LT ${ }^{65}$ & 16 & 1,462 & Outpatient & 69 & 48 & 131 & $24^{c}$ & 32 & 20 & - & 28 & 29 & - \\
\hline SwedeHF ${ }^{48}$ & 23 & 9,640 & Both & 77 & 55 & 133 & 42 & 63 & 56 & 59 & 28 & 28 & 2,018 \\
\hline GWTG-HF 75 & 46 & 18,299 & Inpatient & 82 & 68 & 143 & 44 & 39 & 18 & $1.3^{b}$ & 27 & 39 & 4,104 \\
\hline OPTIMIZE-HF74 & 31 & 10,072 & Inpatient & 76 & 68 & 150 & $32^{c}$ & 32 & - & $1.2^{\mathrm{b}}$ & - & 41 & $537^{d}$ \\
\hline $\mathrm{ADHERE}^{76, \mathrm{e}}$ & 23 & 17,022 & Inpatient & 74 & 69 & 152 & 47 & 32 & 27 & $1.2^{b}$ & 31 & 44 & - \\
\hline TIME-CHF 69 & 18 & 112 & Outpatient & 80 & 64 & 136 & $31^{\mathrm{c}}$ & 43 & 62 & 54 & 27 & 39 & 2,142 \\
\hline CHART-2 ${ }^{70}$ & 62 & 2,154 & Outpatient & 72 & 39 & 128 & $44^{c}$ & 52 & - & 59 & 23 & 34 & $127^{d}$ \\
\hline BIOSTAT-CHF ${ }^{86}$ & 16 & 300 & Outpatient & 78 & 46 & 130 & $33^{f}$ & 50 & 56 & - & - & 36 & 1,559 \\
\hline
\end{tabular}

$\begin{array}{lll}\text { HFrEF } & \text { HFpEF } & \text { Intermediate } \\ \text { characteristics } & \text { characteristics } & \text { characteristics }\end{array}$

Fig. 2 | HFmrEF clinical characteristics and similarities to HFrEF and percentages. AF, atrial fibrillation; CCS, chronic coronary syndrome; CKD, HFpEF in major HF registries. Summary of data from major registries of chronic kidney disease; eGFR, estimated glomerular filtration rate; SBP,

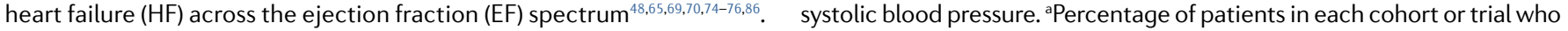

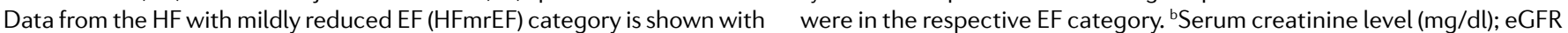
a blue background when resembling data from the HF with reduced EF not provided. clschaemic heart disease as HF aetiology. ${ }^{\mathrm{d}} \mathrm{BNP}$ provided (HFrEF) category, in red when resembling data from the HF with preserved instead of NT-proBNP. eTwo HFrEF categories (EF $<25 \%$ and $25-40 \%$ ), $\mathrm{EF}$ (HFpEF) category and blue/red when intermediate. Values are mean $\mathrm{HFmrEF}$ defined as EF $40-55 \%$ and $\mathrm{HFpEF}$ defined as EF $\geq 55 \%$. ${ }^{\mathrm{f} H} \mathrm{History}$ of (median for N-terminal pro-B-type natriuretic peptide (NT-proBNP)) and

myocardial infarction.

an improved or partially recovered form of $\mathrm{HFrEF}^{65,77}$. In the CHARM programme, most patient characteristics in the HFmrEF group, including age, blood pressure, sex distribution and history of myocardial infarction or atrial fibrillation, resembled those of the HFrEF group $^{72}$. In the SwedeHF registry, the HFmrEF category was more similar to HFpEF for the prevalence of atrial fibrillation and blood pressure levels, but more similar to HFrEF for many other important patient characteristics, such as age and history of chronic kidney disease, diabetes mellitus and IHD ${ }^{48}$. Notably, beyond the crude prevalence of IHD, the adjusted prevalence of IHD was also similar in the HFmrEF and HFrEF groups, and the risk of new ischaemic events was higher in patients with HFmrEF or HFrEF than in those with $\mathrm{HFpEF}^{78}$. The proportion of women was higher in the HFpEF group than in the HFrEF group, with the proportion in the HFmrEF group being more similar to that in the HFrEF group ${ }^{79}$. N-terminal pro-B-type natriuretic peptide (NT-proBNP) levels were similar in the HFmrEF and HFpEF groups and lower than in the HFrEF group ${ }^{48}$, but in the HFmrEF category, the NT-proBNP level was more affected by the presence of confounders such as atrial fibrillation and showed a higher prognostic and discriminatory power than in the HFpEF group ${ }^{80}$. However, in a more extensive and dedicated analysis of 
biomarkers in patients with acute HF, the HFmrEF phenotype was intermediate between the HFpEF and HFrEF phenotypes, with the biomarkers showing differential changes in HFmrEF mostly relating to cardiac stretch and inflammation ${ }^{81}$.

Further studies from SwedeHF have provided additional characterization of HFmrEF versus HFpEF and HFrEF with regard to important comorbidities. Although the prevalence of atrial fibrillation decreased with decreasing EF, the clinical characteristics of patients with atrial fibrillation versus those in sinus rhythm were consistent across the EF spectrum, as was the unfavourable prognostic effect of the presence of atrial fibrillation ${ }^{82}$. Chronic kidney disease and the risk of worsening renal function were more likely to be present in patients with HFpEF but were more strongly associated with mortality in patients with HFmrEF or
$\mathrm{HFrEF}^{83,84}$. This finding potentially suggests that kidney disease develops from the same underlying pathophysiology and in parallel with HFpEF and, therefore, has less prognostic importance than in HFrEF and HFpEF ${ }^{83,84}$. By contrast, in HFmrEF and HFrEF, the presence of kidney disease reflects more severe backward and forward haemodynamic changes in HF and, therefore, has a greater prognostic role ${ }^{83,84}$. Anaemia was more prevalent in patients with HFpEF than in those with HFmrEF or HFrEF, but the presence of anaemia was associated with a similar higher risk of death across the EF spectrum and had a greater association with the risk of death or hospitalization for HF in patients with HFmrEF or HFpEF than in those with HFrEF ${ }^{85}$. In the BIOSTAT-CHF study $^{86}$, the prevalence of other non-cardiac comorbidities in the HFmrEF category was intermediate between the HFrEF and HFpEF categories.

\begin{tabular}{|c|c|c|c|c|c|c|c|c|c|c|c|c|}
\hline Study & $\begin{array}{l}\text { EF type } \\
(\%)^{\mathrm{a}}\end{array}$ & $\begin{array}{l}\text { Number } \\
\text { of patients }\end{array}$ & $\begin{array}{l}\text { Age } \\
\text { (years) }\end{array}$ & $\begin{array}{l}\text { Women } \\
\text { (\%) }\end{array}$ & $\begin{array}{l}\text { SBP } \\
(\mathrm{mmHg})\end{array}$ & CCS (\%) & $\mathrm{AF}(\%)$ & CKD (\%) & $\begin{array}{l}\text { eGFR } \\
(\mathrm{ml} / \mathrm{min} / \\
\left.1.73 \mathrm{~m}^{2}\right)\end{array}$ & $\begin{array}{l}\text { BMI } \\
\left(\mathrm{kg} / \mathrm{m}^{2}\right)\end{array}$ & $\begin{array}{l}\text { Diabetes } \\
\text { mellitus } \\
\text { (\%) }\end{array}$ & $\begin{array}{l}\text { NT-proBNP } \\
(\mathrm{pg} / \mathrm{ml})\end{array}$ \\
\hline \multicolumn{13}{|l|}{ HFrEF } \\
\hline BB-meta-HF ${ }^{99, b, c}$ & 93 & 13,443 & $\begin{array}{l}61 / 63 / \\
63 / 64\end{array}$ & $\begin{array}{l}20 / 23 / \\
25 / 27\end{array}$ & $\begin{array}{l}114 / 120 / \\
127 / 130\end{array}$ & $\begin{array}{l}58 / 66 / \\
69 / 81\end{array}$ & 0 & - & $\begin{array}{l}62 / 61 / \\
66 / 65\end{array}$ & 27 & $\begin{array}{l}25 / 26 / \\
24 / 22\end{array}$ & - \\
\hline $\mathrm{DIG}^{159}$ & 75 & 5,874 & 63 & 21 & 125 & $65^{d}$ & 0 & - & $1.3^{\mathrm{e}}$ & 27 & 28 & - \\
\hline $\begin{array}{l}\text { PARADIGM- } \\
\text { PARAGON }\end{array}$ & 63 & 8,399 & $\begin{array}{l}61 / 63 / \\
66\end{array}$ & $\begin{array}{l}19 / 21 / \\
24\end{array}$ & $\begin{array}{l}117 / \\
121 / \\
124\end{array}$ & $\begin{array}{l}39 / 44 / \\
45^{d}\end{array}$ & $\begin{array}{l}28 / 33 / \\
45\end{array}$ & - & $\begin{array}{l}68 / 68 / \\
67\end{array}$ & $\begin{array}{l}27 / 28 / \\
29\end{array}$ & $\begin{array}{l}34 / 34 / \\
35\end{array}$ & $\begin{array}{l}2,183 / \\
1,645 / \\
1,406\end{array}$ \\
\hline
\end{tabular}

\begin{tabular}{|c|c|c|c|c|c|c|c|c|c|c|c|c|}
\hline \multicolumn{13}{|l|}{ HFmrEF } \\
\hline TOPCAT $^{87}$ & 15 & 520 & 66 & 37 & 128 & $44^{d}$ & - & - & 70 & 31 & 29 & - \\
\hline BB-meta-HF ${ }^{99, b}$ & 5 & 575 & 71 & 34 & 131 & 91 & 0 & - & 66 & 27 & 24 & - \\
\hline $\mathrm{CHARM}^{72}$ & 17 & 1,322 & 65 & 30 & 130 & $58^{d}$ & 26 & - & $1.2^{\mathrm{e}}$ & 28 & 29 & - \\
\hline $\begin{array}{l}\text { PARADIGM- } \\
\text { PARAGON }\end{array}$ & 11 & 1,427 & 71 & 40 & 131 & $32^{d}$ & 34 & - & 65 & 30 & 44 & 1,070 \\
\hline
\end{tabular}

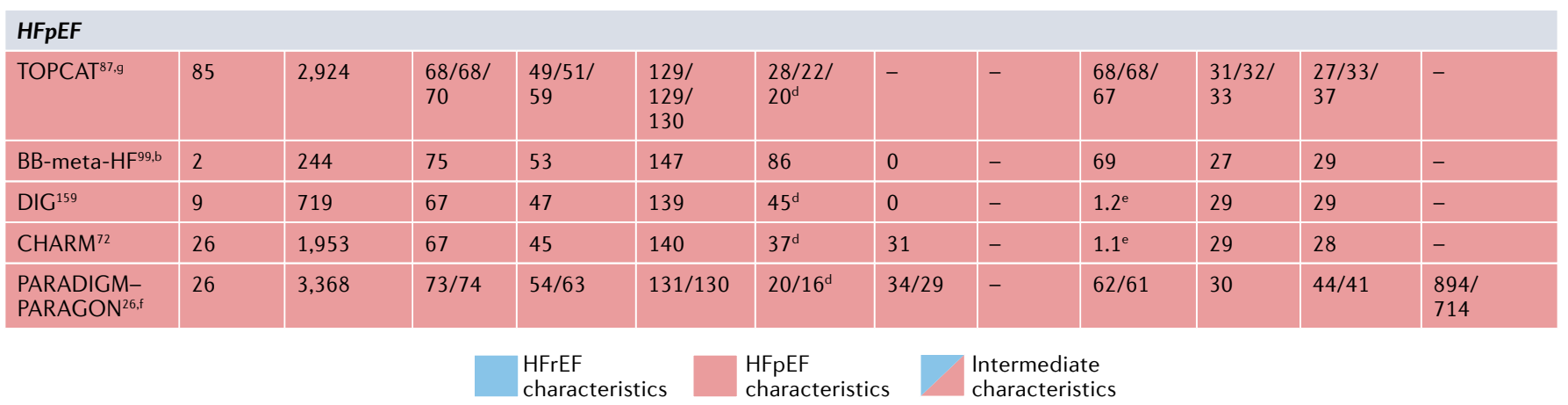

Fig. 3 | HFmrEF clinical characteristics and similarities to HFrEF and HFpEF in major RCTs of HF. Summary of data from randomized clinical trials (RCTs) of heart failure (HF) across the ejection fraction (EF) spectrum ${ }^{26,72,87,99,159}$. Data from the HF with mildly reduced EF (HFmrEF) category is shown in blue when resembling the $\mathrm{HF}$ with reduced $\mathrm{EF}$ (HFrEF) category, in red when resembling the HF with preserved EF (HFpEF) category and blue/red when intermediate. Values are mean, median or percentage, as reported in the original trial publications. AF, atrial fibrillation; CCS, chronic coronary syndrome; CKD, chronic kidney disease; eGFR, estimated glomerular filtration rate; NT-proBNP,
$\mathrm{N}$-terminal pro-B-type natriuretic peptide; SBP, systolic blood pressure. a Percentage of patients in each cohort or trial who were in the respective EF category. ${ }^{\mathrm{b} P a t i e n t s}$ in sinus rhythm. ${ }^{\mathrm{C}}$ Four $\mathrm{HFrEF}$ categories (EF $<20 \%$, 20-25\%, 26-34\% and 35-39\%). ${ }^{\mathrm{d}} \mathrm{History}$ of myocardial infarction. ${ }^{\mathrm{e} S e r u m}$ creatinine level (mg/dl); eGFR not provided. ${ }^{\mathrm{f}} \mathrm{EF}$ range $\leq 42.5 \%$ for HFrEF, $>42.5-52.5 \%$ for HFmrEF and $>52.5 \%$ for HFpEF (chosen to avoid bias towards $E F$ values ending in 0 or 5 ); three $\mathrm{HFrEF}$ categories ( $E F \leq 22.5 \%$, 22.5-32.5\% and $>32.5-42.5 \%$ ) and two HFpEF categories (EF $>52.5-62.5 \%$ and $>62.5 \%$ ). ${ }^{9}$ Three HFpEF categories (EF 50.00-54.99\%, 55.00-59.99\% and $\geq 60.00 \%$ ). 
The BMI in the HFmrEF group was intermediate between that of the HFrEF and HFpEF groups in European registries as well as in the CHARM programme ${ }^{48,65,72}$. By contrast, in the TOPCAT and PARAGON-HF trials, the BMI of the group of patients with HFmrEF resembled more the BMI of the HFpEF group and was consistently $\geq 30 \mathrm{~kg} / \mathrm{m}^{2}$ as a mean or median ${ }^{26,87}$. In a US community study and in few dedicated RCTs of HFpEF, obesity was predominant among patients with HFpEF, and the mean BMI was $>35 \mathrm{~kg} / \mathrm{m}^{2}\left(\mathrm{REFS}^{88-90}\right)$. The complex interaction between obesity and HF remains only partially understood. Obesity is a risk factor for HF but potentially more strongly so for HFpEF than for $\mathrm{HFrEF}^{90-93}$, whereas obesity has not been investigated specifically as a risk factor for $\mathrm{HFrEF}^{86}$. Secondary mitral regurgitation and right ventricular dysfunction have been reported to be less prevalent in HFmrEF and HFpEF than in HFrEF, whereas data on the epidemiology of secondary tricuspid regurgitation by EF phenotype are currently lacking ${ }^{94,95}$. Differences in HFmrEF characteristics according to race or ethnicity are also poorly studied. In a US cohort of patients with HFmrEF, Black patients had a lower prevalence of previous myocardial infarction and Hispanic patients had more comorbidities but better survival compared with the other ethnicities ${ }^{96}$.

Previous research has also assessed the degree of neurohormonal activation across the EF spectrum, showing higher circulating neurohormone levels in patients with HFrEF than in those with HFmrEF or HFpEF ${ }^{80,97,98}$. The higher neurohormonal activation in HFrEF might reflect the greater HF severity and be a marker of higher rates of cardiovascular events in patients with HFrEF than in patients with HFmrEF or HFpEF, as well as the observed efficacy of neurohormonal inhibition in patients with HFrEF, and potentially in those with HFmrEF, but not in patients with HFpEF (as discussed below) ${ }^{26,72,99}$.

Although HFmrEF is overall more similar to HFrEF than HFpEF for most clinical characteristics and for treatment response, and might therefore be interpreted as being a mild form of HFrEF (that is, as HF with mildly reduced $\mathrm{EF})^{19,20}$, some inconsistencies are found across different analyses. However, in all the studies reported above, one consistent observation is that IHD has a similar prevalence in HFmrEF and HFrEF, which is greater than in HFpEF. From the perspective of research and clinical trial design, considering all these nuances would be beneficial. From the perspective of routine clinical care, a more simplistic message might be most effective, namely that, taken together, most data suggest that HFmrEF more closely resembles the HFrEF phenotype than the HFpEF phenotype (FIGS 1-3).

\section{Prognosis}

Accurate prognostication in HFmrEF is important on a population level for proper resource allocation and for power and sample size assessments for trials. At the individual level, accurate prognosis is important for patients, families and providers to have reasonable expectations and make treatment decisions and prioritizations accordingly.

In observational and large registry studies, the risk of adverse cardiovascular outcomes seems to be lower in patients with HFmrEF or HFpEF than in patients with $\mathrm{HFrEF}^{46,65}$. By contrast, consistent with the higher age and the presence of comorbidities, the risk of non-cardiovascular events seems to be greater in patients with HFpEF, and possibly those with HFmrEF, than in patients with $\mathrm{HFrEF}^{46,65}$. All-cause mortality is higher in HFrEF than in HFmrEF and HFpEF in some reports $^{48,49,65,68,70}$, lower in HFmrEF than in HFpEF and HFrEF in other reports ${ }^{26,100}$, and similar across the EF spectrum in others ${ }^{74,75,101}$. An analysis reported in 2020 of a large data set with available echocardiography data suggested a U-shaped relationship between EF and mortality, with very high values of $\mathrm{EF}(\mathrm{EF}>65 \%)$ also associated with increased mortality after adjustment for conditions such as HF, mitral regurgitation, increased wall thickness and anaemia ${ }^{49}$. This observation might build the ground for a further EF subtype that might be defined as HF with supra-normal EF (HFsnEF) ${ }^{49}$. The enrolment of exclusively, or of different proportions of, inpatients versus outpatients in these studies might partly explain the heterogeneous data.

Differences in outcomes across the EF spectrum have been observed in both real-world studies and RCTs. The differences are greater in RCTs, in which patients with HFpEF or HFmrEF had a considerably lower risk of cardiovascular events than patients with HFrEF (FIG. 4). RCTs are more selective than real-world studies; for example, in RCTs, patients have a younger age and fewer comorbidities but more severe $\mathrm{HF}$ as a result of an enrichment strategy. In the CHARM programme ${ }^{72}$, the risk of all-cause death over approximately 3 years of follow-up was $15.8 \%$ in patients with HFmrEF, which is remarkably low compared with estimates from registries $(7-34 \% \text { at } 1 \text { year })^{48,65,69,70,75}$. Additionally, patients with HFmrEF or HFpEF had a lower risk of death or hospitalization for HF than patients with $\mathrm{HFrEF}^{72}$. When the associations with EF were analysed as a continuous spline variable, a steep decrease in rates was observed with increasing EF until an EF of 50\% for all-cause and cardiovascular death and $40 \%$ for the risk of hospitalization for HF, after which the incidence rate curves flattened ${ }^{72}$. The risk of non-cardiovascular adverse outcomes was overall higher in the HFpEF population $^{72}$. By contrast, in the TIME-CHF trial ${ }^{69}$, similar hospital admission rates and mortality were observed regardless of EF.

Prognostic data also differ in incident versus prevalent HF. In one study, the rates of all-cause death were 497 per 10,000 person-years for incident HFmrEF versus 394 per 10,000 person-years for incident HFpEF and 459 per 10,000 person-years for incident HFrEF, suggesting worse survival in patients with incident $\mathrm{HFmrEF}$ or HFrEF than in those with incident $\mathrm{HFpEF}^{64}$. These data are important because they more closely represent a 'pure' HFmrEF, that is, new onset of HFmrEF, whereas in most of the cross-sectional analyses of HF cohorts, the proportion of patients with HFmrEF with transitioning EF might be relevant and confound the true prognostic influence of HFmrEF. Notably, in this study, the similar all-cause mortality in HFmrEF and HFrEF, which was higher than in HFpEF, which was reported after adjustment for several patient characteristics, including 

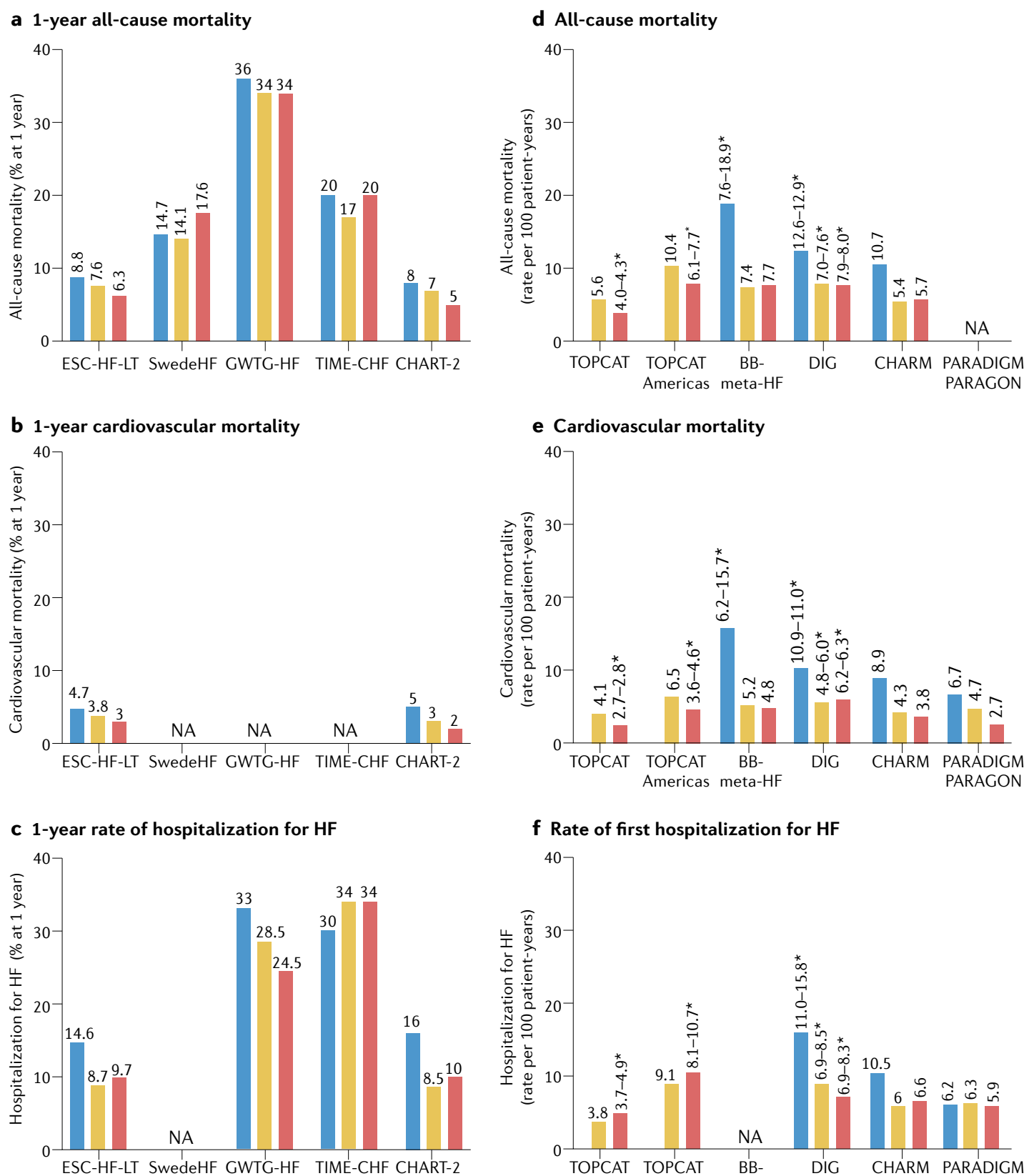

f Rate of first hospitalization for $\mathrm{HF}$

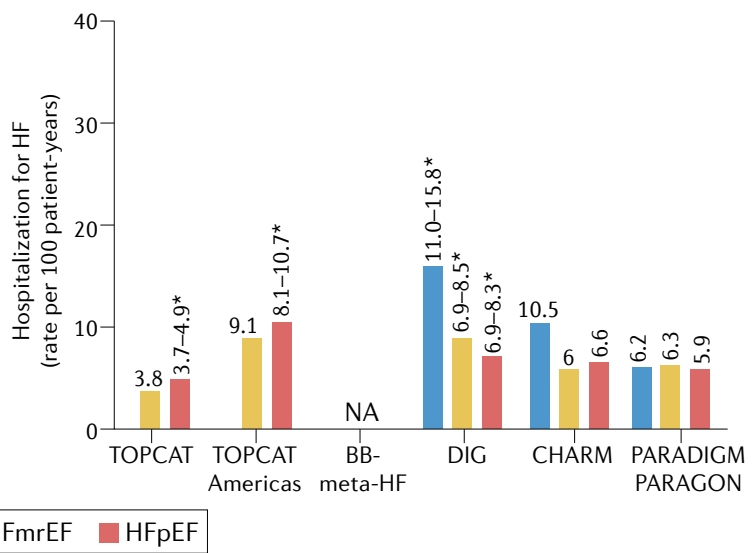

history of myocardial infarction and coronary heart disease, suggests that in terms of pathophysiology, HFmrEF might be closer to HFrEF than to HFpEF. Another explanation might be that in most of the studies, the observation that patients with HFpEF have a similar or worse prognosis than patients with HFmrEF or HFrEF might be due to HFpEF having a more gradual onset and longer disease duration, which leads to later diagnosis and worse prognosis at enrolment ${ }^{64}$.

Composite prognostic models and scores are supporting tools in the risk stratification of patients with HF. The most-used risk scores, such as the MAGGIC score $^{102}$, the Seattle HF model (SHFM) ${ }^{103}$ and the more contemporary PREDICT-HF ${ }^{104}$, have been validated. However, these scores have been derived predominantly (MAGGIC score) or exclusively (SHFM and PREDICT-HF scores) in patients with HFrEF. The peak $\mathrm{VO}_{2}$, an integrated parameter from the cardiopulmonary exercise test, and the Heart Failure Survival Score (HFSS) were both developed primarily for transplantation and mechanical circulatory support selection in patients with advanced (generally reduced EF) HF, but also seem to predict prognosis in patients with $\mathrm{EF}$ $>40 \%{ }^{105}$. No specific risk models for prognostication in HFmrEF are currently available, although reports from the SwedeHF registry supported the use of MAGGIC 
4 Fig. 4 | Outcomes according to EF in patients with HF in major registries and RCTs. All-cause mortality (panels a,d), cardiovascular mortality (panels b,e) and hospitalization for heart failure (HF) (panels c,f) according to left ventricular ejection fraction (EF) in patients with $\mathrm{HF}$ in major registries and randomized clinical trials (RCTs). Data from REFS $26,48,65,69,70,72,75,87,99,159$. ESC-HF-LT ${ }^{65}$ included prevalent and incident HF; 1 -year rates are shown for all outcomes. SwedeHF ${ }^{48}$ included prevalent and incident HF; 1-year rates of all-cause death are shown; no data on cardiovascular mortality or hospitalization for $\mathrm{HF}$ were available. GWTG-HF75 included in-hospital new-onset and worsening HF; 1-year all-cause mortality and 1-year hospitalization for HF estimated from the 1-year time point on the $x$-axis and percentage on the $y$-axis of Kaplan-Meier curves in REF. ${ }^{75}$; data on 5-year all-cause mortality and 5-year rate of hospitalization for HF shown in table 3 of the original article; no data on cardiovascular mortality were available. TIME-CHF ${ }^{69}$ included prevalent HF; rate of 1-year hospitalization for HF or death is shown; rates estimated from the 1-year time point on the $x$-axis and percentage on the $y$-axis of Kaplan-Meier curves in REF. ${ }^{69}$; data on all-cause mortality and rate of hospitalization for HF during the overall follow-up reported in the main text of the original article; no data on cardiovascular mortality were available. CHART-2 (REF. $\left.{ }^{70}\right)$ included prevalent and incident HF; 1-year rates for all outcomes estimated from the 1-year time point on the $x$-axis and percentage on the $y$-axis of Kaplan-Meier curves in REF. ${ }^{70}$. TOPCAT and TOPCAT Americas trials ${ }^{87}: 1$-year rates are shown for all outcomes, patients with $E F \geq 50 \%$ were divided into subcategories (50.00-54.99\%, 55.00-59.99\% and $\geq 60.00 \%$ ); we reported the ranges of event rates if different across subcategories (asterisks); event rates for patients with $\mathrm{EF}<50 \%$ are provided in table 3 of $\mathrm{REF}^{.7}$. BB-meta- $\mathrm{HF}^{99}$ : rates at a median follow-up of 1.3 years are shown for all outcomes in patients in sinus rhythm; patients with $E F<40 \%$ were divided into subcategories (<20\%, 20-25\%, 26-34\% and 35-39\%); we reported the ranges of event rates if different across subcategories (asterisks); event rates in the $40-49 \%$ and $\geq 50 \%$ categories were directly provided in the trial publication, and we converted them into an estimated event rate per 100 patient-years by dividing by 1.3 ; data on the rate of first hospitalization for HF were not provided. DIG ${ }^{159}$ : 1 -year rates shown for all outcomes; we reported the ranges of event rates if different between the treatment and placebo groups (asterisks). CHARM $^{72}$ : 1-year rates provided for all outcomes. PARADIGM-HF-PARAGON-HF ${ }^{26}$ : no global follow-up duration was provided in the trial publication; therefore, we estimated rates per 100 patient-years by dividing by 2.25 (the median follow-up duration of PARADIGM-HF) for the EF $<40 \%$ category and by dividing by 2.9 (the median follow-up duration of PARAGON-HF) for the EF $40-49 \%$ and $E F \geq 50 \%$ categories; data on all-cause mortality were not provided. HFmrEF, heart failure with mildly reduced ejection fraction; $\mathrm{HFpEF}$, heart failure with preserved ejection fraction; HFrEF, heart failure with reduced ejection fraction; NA, not available.

and SHFM in the general population of patients with $\mathrm{HF}$, regardless of $\mathrm{EF}^{106,107}$.

Secondary mitral regurgitation is related to the degree of left ventricular dilatation and EF, but seems to be associated with poor outcome regardless of EF and has a potentially greater prognostic role in HFmrEF than in $\mathrm{HFrEF}^{94,108}$. By contrast, the prognostic role of secondary tricuspid regurgitation in HFmrEF is currently unexplored. Right ventricular dysfunction was shown to be a prognostic predictor of poor outcomes in HF regardless of $\mathrm{EF}^{95}$.

One of the less explored aspects of HFmrEF is the risk of life-threatening ventricular arrhythmias. Sudden cardiac death (SCD) is a feared yet often preventable mode of death in patients with HFrEF. The risk stratification for SCD in HF is based on the degree of reduced $\mathrm{EF}^{109}$. The risk of SCD is not considered to be high in patients with $\mathrm{EF}>40 \%$. In HFmrEF and HFpEF, the mode of death, and in particular, SCD, has been poorly reported or imprecisely adjudicated in observational studies and RCTs, frequently confounded by the competing risk of non-cardiovascular deaths. However, when the mode of death was available, SCD accounted for $30-40 \%$ of the cardiovascular modes of death and should not be neglected $^{110}$. Therefore, risk stratification for SCD might be as relevant in HFpEF and HFmrEF as in HFrEF.
However, available prediction models for SCD, such as the Seattle proportional risk model and others, have been derived from cohorts that included almost exclusively patients with $\mathrm{EF}<40 \%$ and are therefore not applicable to patients with HFmrEF or HFpEF ${ }^{111-113}$. Alternative emerging methods, such as cardiac MRI, might provide further information for risk quantification ${ }^{114}$.

Incident comorbidities in patients with $\mathrm{HF}$ and factors precipitating worsening HF can have a strong effect on disease progression and the risk of hospitalization and death. The precipitants of incident and worsening HFmrEF resemble those of HFpEF, with a single, essential exception: IHD $^{67,115}$. In SwedeHF ${ }^{78}$, IHD was more common in HFmrEF and HFrEF and also had a greater adverse prognostic influence in HFmrEF and HFrEF than in HFpEF. However, other analyses reported similar detrimental effects of IHD on survival in HFpEF to those in the other EF phenotypes of HF ${ }^{116-119}$.

Prognostication in HF relies on multiple factors. Circulating levels of natriuretic peptides were shown to be associated with morbidity and mortality in both HFpEF and HFrEF ${ }^{80,120-122}$. Studies from the SwedeHF registry also support the prognostic role of natriuretic peptides in $\mathrm{HFmrEF}^{68,69,80,120}$, even suggesting a greater prognostic and discriminatory role for NT-proBNP in HFmrEF, by showing a greater association between continuous NT-proBNP levels and outcomes as well as higher areas under the receiver operating characteristic curve for death and death or hospitalization for HF in HFmrEF than in the other EF categories ${ }^{80}$. In the heterogeneous setting of HFmrEF, which includes patients who had incident HFmrEF and those with transitioning $\mathrm{EF}$ (deteriorating or improving), natriuretic peptide levels might be a more reliable marker of severity than the EF itself. Indeed, in a prospective cohort study, the difference in mortality across the three HF phenotypes vanished after adjustment for NT-proBNP levels, which might imply that although natriuretic peptide levels and cardiovascular risk are lower in patients with HFpEF or HFmrEF than in patients with HFrEF, at a given value of NT-proBNP, the risk of death might be similar regardless of $\mathrm{EF}^{68,80}$. In TIME-CHF ${ }^{69}$, a significant potential benefit of NT-proBNP-guided therapy was similarly observed in HFrEF and HFmrEF but not in HFpEF.

\section{Phenotyping HFmrEF beyond EF}

Owing to the limitations of the EF construct and measurement, numerous alternative methods are advocated for and are emerging to phenotype HF. Myocardial tissue characterization by cardiac MRI is part of the routine diagnostic work-up in patients with HF of unknown aetiology ${ }^{123}$. Emerging studies also highlight the importance of cardiac MRI in patients with EF $>40 \%{ }^{114,124,125}$. Late gadolinium enhancement (LGE) is known to be a major prognostic marker and a validated predictor of SCD and life-threatening arrhythmias in HFrEF ${ }^{126}$. However, LGE also correctly classifies the risk of death and SCD in patients with mild-to-moderate reduction in $\mathrm{EF}^{114,127}$, and therefore could be considered to identify patients at increased risk of SCD who are suitable for trials that test strategies for primary prevention of SCD regardless of the $\mathrm{EF}^{114}$. 
The assessment of myocardial strain and in particular global longitudinal strain (GLS) from speckle-tracking analysis of $2 \mathrm{D}$ echocardiography is an emerging technique complementary to EF for the quantification of systolic and diastolic ventricular and atrial function ${ }^{128,129}$. Measurement of GLS has revealed how systolic dysfunction (reduced GLS) can be present even if the EF is preserved or normal ${ }^{130,131}$. Large observational studies and meta-analyses also support an incremental and independent role for GLS beyond EF in terms of mortality prediction in patients with $\mathrm{EF}>35 \%{ }^{132,133}$.

In the era of precision medicine, deep aetiological characterization of HF becomes both possible and meaningful to maximize the understanding of the underlying disease, predict its natural progression, individualize treatment strategies, and identify and design trials for novel treatment targets. Biomarkers and proteomic signatures might further help to highlight differences across the EF categories. A multiple biomarker approach in acute HF demonstrated that patients with HFmrEF have an intermediate profile between HFrEF (cardiac stretch) and HFpEF (inflammation) ${ }^{81}$. Proteomic variability was high across the EF phenotypes, and the mid-range category was heterogeneous and resembled HFpEF more than $\mathrm{HFrEF}^{134}$.

Cardiac amyloidosis and hypertrophic cardiomyopathy are two important examples of how rigorous diagnostics and disease characterization have helped in both identifying patients for disease-targeted therapies and excluding patients from trials in which the target of therapy is different, such as HFpEF trials. Prevalent IHD modified the exposure to further ischaemic events but also non-ischaemic events in patients with HFmrEF enrolled in the SwedeHF registry ${ }^{78}$. Among non-ischaemic aetiologies of HF, specific phenotypes such as sarcoidosis might portend increased risks of poor outcomes irrespective of the severity of left ventricular dysfunction ${ }^{135}$. The genetic background of non-severely reduced $\mathrm{EF}$ in some cases can lead to alternative strategies to protect patients with a high risk of SCD even in the absence of reduced EF. Patients with specific genotypes of dilated cardiomyopathy, including variants in the gene encoding lamin $\mathrm{A} / \mathrm{C}$ and variants in genes encoding desmosomal proteins, had a lower survival free from potentially fatal ventricular arrhythmias despite having $\mathrm{EF}>35 \%$, which is the guideline-based threshold for receiving an implantable cardioverter-defibrillator (ICD) $)^{136,137}$.

\section{Transitioning through HFmrEF}

A major limitation of registry, cohort and trial data sets is the relative paucity of longitudinal data. In studies of prevalent HF, a single assessment of EF provides a static snapshot of something that entails measurement error and often shifts over time. However, EF is subject to change owing to effects of therapy or the natural progression of $\mathrm{HF}^{138,139}$. Some patients with HFmrEF might be in transition from preserved to reduced $\mathrm{EF}$ as a result of an acute event (such as an ischaemic event) and, conversely, other patients with HFmrEF might be recovering from reduced to preserved $\mathrm{EF}$ after medical or device therapy for HF or invasive anti-ischaemic treatments ${ }^{78,115,117,118,122}$.
In one prospective study of 126 patients with $\mathrm{HFpEF}$ with baseline EF of $63 \pm 8 \%$ who were followed up for $>11$ years, $9.5 \%$ of patients progressed to HFmrEF and only $1.6 \%$ to HFrEF, suggesting that in patients with HFpEF, the EF is likely to remain stable over time (that is, $\mathrm{EF} \geq 50 \%)^{118}$. Similarly, of 100 patients hospitalized with HFpEF (baseline EF of $67 \pm 9 \%$ ) who underwent EF assessment $\geq 1$ year after hospital discharge, $11 \%$ had a decline to HFmrEF over a mean follow-up of 31.5 months ${ }^{140}$. In both studies, most patients transitioning to HFmrEF from HFpEF had an EF of 50-55\% at the baseline. This finding might explain the efficacy of sacubitril-valsartan in patients with an EF within the lower range of HFpEF shown in the PARAGON-HF trial $^{26,59}$, and might also call into question whether the EF range 50-55\% most appropriately applies to HFmrEF or to HFpEF. In a retrospective analysis of 4,942 patients in SwedeHF, roughly $33 \%$ of patients with HFmrEF or HFpEF transitioned to a lower EF category and $25 \%$ of those with HFmrEF or HFrEF transitioned to a higher EF category over a median follow-up of 1.4 years (interquartile range $0.5-3.0$ years) ${ }^{122}$ (FIG. 5). Among patients with HFmrEF, $37 \%$ and $25 \%$ switched to HFrEF and HFpEF, respectively, whereas among patients with HFrEF or HFpEF, $16 \%$ and $21 \%$, respectively, switched to $\mathrm{HFmrEF}^{122}$. Several factors were associated with increasing EF (female sex, atrial fibrillation and less severe $\mathrm{HF}$ ) or decreasing EF (diabetes, IHD and more severe HF) ${ }^{122}$. This registry-based analysis reflects EF assessment as performed in daily clinical care, with a bias towards obtaining repeat EF measurements in patients who reported a clinical deterioration or were judged to be sicker. Therefore, the risk of EF declining over time might have been overestimated. In the Olmsted County (Minnesota, USA) cohort, 39\% of patients crossed from HFpEF (EF $250 \%)$ to HFrEF (EF $<50 \%)$ and $39 \%$ of patients crossed from HFrEF to HFpEF over a 5-year observation period $^{23}$.

In HFrEF, evidence-based drugs seem to improve EF only by a few percentage points on average ${ }^{139,141,142}$. Growing evidence suggests that HF with recovered EF, or HF with improved EF, is frequent and might represent a further phenotype characterized by less myocardial damage and associated with a better outcome than $\mathrm{HF}$ with persistently reduced $\mathrm{EF}$, although with a risk of EF reduction recurrence ${ }^{122,138}$. The recovery of EF from reduced to preserved EF has been shown to be associated with a $45 \%$ reduced risk of all-cause death or hospitalization for HF in a study from the SwedeHF registry ${ }^{122}$. Among 1,057 consecutive patients with HF with available baseline and 1-year measurements of EF, patients with recovered EF had better outcomes in terms of cardiovascular death and hospitalization for HF than those in either the HFpEF or the HFrEF subgroup ${ }^{143}$. Similar findings were observed in a retrospective analysis of $>2,000$ outpatients with $\mathrm{HF}^{77}$. In an analysis from the SwedeHF registry, increasing EF was associated with a lower risk of all-cause death or hospitalization for HF, whereas decreasing EF was associated with a higher risk $^{122}$ (FIG. 5). Partial or complete recovery of HFrEF was associated with significantly better outcomes than stable EF in HFrEF and, conversely, deterioration from 


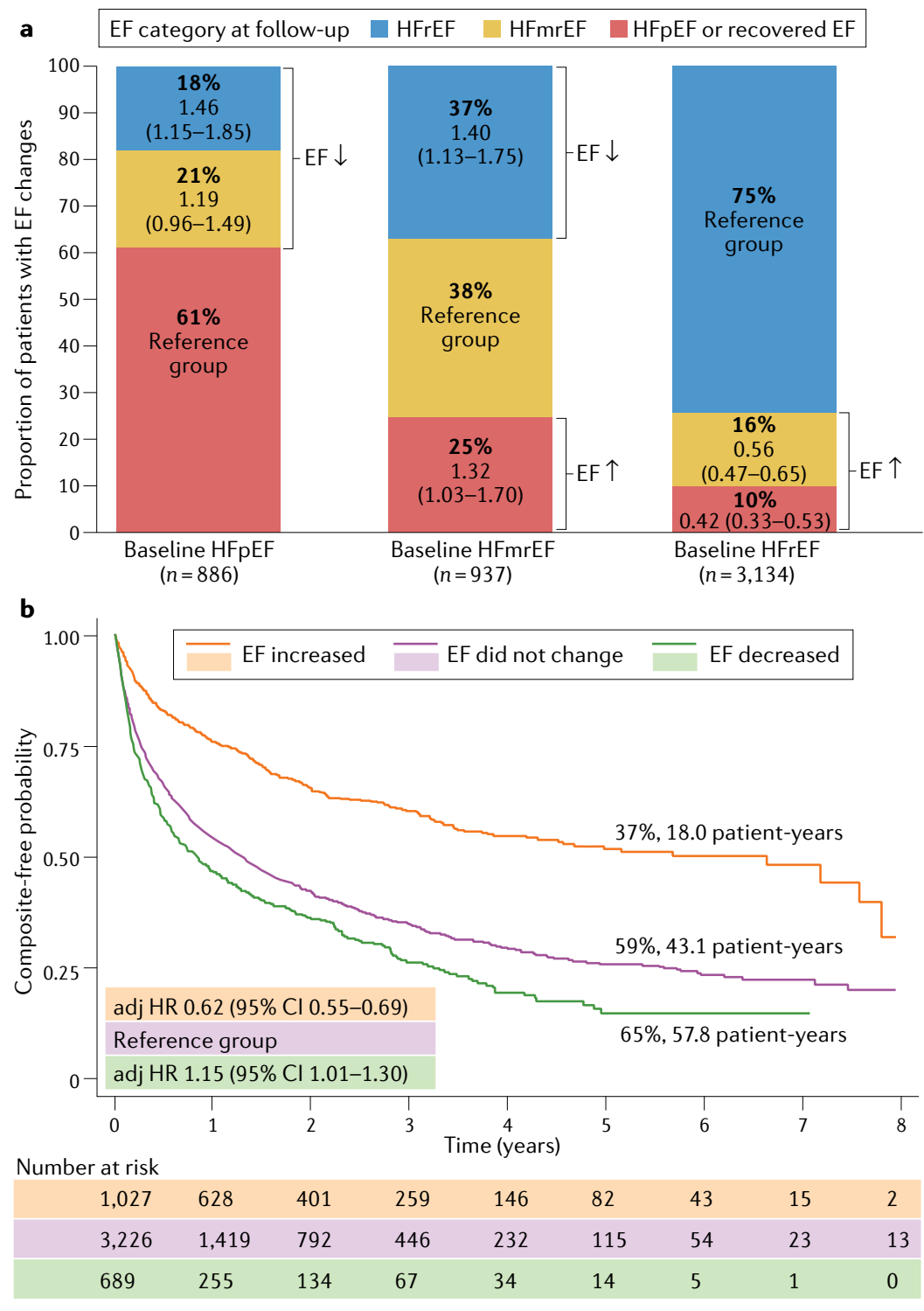

Fig. 5 | Trajectories and changes in EF and outcomes over time in patients with HF. Data from a retrospective, nationwide, registry study of patients with heart failure (HF) ${ }^{122}$. a | Each bar segment shows the proportion of patients with changes in left ventricular ejection fraction (EF) at follow-up and the associated hazard ratio (HR) and $95 \%$ confidence interval $(\mathrm{Cl})$ of all-cause death or hospitalization for HF by EF category at baseline. In each category, the group of patients with a stable EF over time was used as reference. $\mathbf{b}$ | The risk of all-cause death or hospitalization for $\mathrm{HF}$ relative to $\mathrm{EF}$ changes. adj, adjusted; HFmrEF, heart failure with mildly reduced ejection fraction; HFpEF, heart failure with preserved ejection fraction; HFrEF, heart failure with reduced ejection fraction. Parts $\mathbf{c}$ and $\mathbf{d}$ adapted with permission from REF. ${ }^{122}$, Elsevier.

HFpEF or HFmrEF to HFrEF was associated with worse prognosis than stable $\mathrm{EF}$ in $\mathrm{HFrEF}^{122}$. In a retrospective cohort study focusing on HFmrEF, only $15 \%$ of patients had stable HFmrEF, as assessed by comparing with previous echocardiography exams ${ }^{144}$. The risk of all-cause death, cardiovascular death and hospitalization for HF was higher for those with EF decreasing from $>50 \%$ than in those with $\mathrm{EF}$ improving from $<40 \%$, after adjustment for confounding factors. Interestingly, the prognosis of patients with improving EF was not better but similar to that in patients with stable mid-range EF, suggesting that stable HFmrEF might represent a relatively low-risk phenotype $^{144}$. Changes in EF in patients with HF have been observed to be more likely to occur in the early stages of the disease, following diagnosis and treatment initiation, and are less pronounced in patients with HFmrEF than in those with $\mathrm{HFrEF}^{145}$. However, EF trajectories in patients with diagnosed HFmrEF or HFrEF showed an inverse $U$ shape, with EF tending to decline over time in both groups and finally overlap at 10 years of follow-up ${ }^{145}$.

\section{Treatment of HFmrEF}

Historically, neurohormonal antagonist drugs (reninangiotensin system (RAS) inhibitors, $\beta$-blockers and mineralocorticoid-receptor antagonists (MRAs)) have been the cornerstone of pharmacological therapy for patients with HFrEF. In 2014, angiotensin-receptor blocker-neprilysin inhibitors (ARNIs), and in 2019, sodium-glucose cotransporter 2 (SGLT2) inhibitors, both neurohormonal modulators, were also shown to be effective in $\mathrm{HFrEF}^{6}$. However, in subsequent trials, RAS inhibitors, $\beta$-blockers, MRAs and ARNIs were overall not effective in patients with HFpEF, generally defined as $E F \geq 40 \%$ or $\geq 45 \%{ }^{51,57-59,146,147}$. Sotagliflozin, an SGLT2-SGLT1 inhibitor, was effective in patients with type 2 diabetes mellitus and HF across the EF spectrum $^{60}$, and dedicated trials of empagliflozin ${ }^{148,149}$ and dapagliflozin ${ }^{53,150}$ in HFmrEF and HFpEF are ongoing. No dedicated interventional trial has addressed HFmrEF specifically. Observational data are scarce and do not prove efficacy, but can provide hints regarding interventions that might potentially be beneficial in HFpEF and HFmrEF. With the known limitations of observational studies, large, registry-based studies suggest that angiotensin-converting enzyme inhibitors, angiotensin-receptor antagonists and $\beta$-blockers might potentially provide benefit in patients with $\mathrm{HFmrEF}^{10,151}$. The rate of HFrEF medication use in registries is high in the population of patients with HFmrEF, which might be explained by the role of these therapies in treating risk factors and comorbidities that are frequent in $\mathrm{HF}$ regardless of EF, such as hypertension, diabetes, chronic kidney disease, IHD and atrial fibrillation ${ }^{48,152}$. Diuretic use is also high in patients with HFmrEF, presumably for symptom relief, which is indicated regardless of $\mathrm{EF}^{6,48}$. These findings might also suggest that in clinical practice, patients with HFmrEF are frequently treated like those with HFrEF or, alternatively, that a large proportion of these patients have improved from HFrEF, in which case therapy should, and generally does, continue $48,65,69,70,75,153,154$.

Most RCTs in HFpEF had EF cut-off values of $40 \%$ or $45 \%$ and therefore included, either completely or partially, the HFmrEF category ${ }^{51,57-59]}$ (TABLE 1; FIG. 6). In the CHARM programme ${ }^{72}$, therapy with candesartan reduced the risk of the composite of cardiovascular death and hospitalization for HF in patients with HFrEF or HFmrEF but not in patients with HFpEF. Spline analyses, in which EF was analysed as a continuous variable, clearly showed that candesartan efficacy was constant with lower EF but started to decline when the EF increased above $50 \%^{72}$. However, even in the HFpEF 
Table 1 | Major phase III randomized, controlled trials including patients with HF with EF of 40-49\%

\begin{tabular}{|c|c|c|c|c|c|c|c|c|c|}
\hline Study & Year & $\begin{array}{l}\text { Number } \\
\text { of } \\
\text { patients }\end{array}$ & Drug & Class & $\begin{array}{l}\text { EF } \\
\text { range } \\
(\%)\end{array}$ & $\begin{array}{l}\text { Main HF } \\
\text { category } \\
\text { focus }\end{array}$ & Primary outcome & $\begin{array}{l}\text { Follow-up } \\
\text { (months) }\end{array}$ & Refs \\
\hline ANZ & 1997 & 415 & Carvedilol & $\beta$-Blocker & $<45$ & HFrEF & $\begin{array}{l}\text { Death, hospitalization for } \\
\text { HF or worsening HF }\end{array}$ & 19 & 165 \\
\hline CHARM-Preserved & 2003 & 3,023 & Candesartan & ARB & $>40$ & HFpEF & $\begin{array}{l}\text { Cardiovascular death or } \\
\text { first hospitalization for HF }\end{array}$ & 37 & 51 \\
\hline SENIORS & 2005 & 2,135 & Nebivolol & $\beta$-Blocker & All & HFrEF & $\begin{array}{l}\text { All-cause mortality, } \\
\text { hospitalization for } \\
\text { cardiovascular causes }\end{array}$ & 21 & 147 \\
\hline PEACE & 2004 & 8,290 & Perindopril & ACEi & $>40$ & HFpEF & $\begin{array}{l}\text { Cardiovascular mortality, } \\
\text { Ml or revascularization }\end{array}$ & 58 & 166 \\
\hline PEP-CHF & 2006 & 850 & Perindopril & ACEi & $\begin{array}{l}\text { LVWMI } \\
1.4-1.6\end{array}$ & $\begin{array}{l}\text { HFmrEF } \\
\text { and } \\
\text { HFpEF }\end{array}$ & $\begin{array}{l}\text { All-cause mortality, } \\
\text { hospitalization for HF }\end{array}$ & 26 & 146 \\
\hline MIRACLE-EF & 2012 & 44 & $\begin{array}{l}\text { CRT } \\
\text { pacemaker }\end{array}$ & Devices & $36-50$ & HFmrEF & $\begin{array}{l}\text { Death or first } \\
\text { hospitalization for HF }\end{array}$ & $\begin{array}{l}\text { Stopped } \\
\text { early }\end{array}$ & 168 \\
\hline TOPCAT & 2014 & 3,445 & Spironolactone & MRA & $\geq 45$ & HFpEF & $\begin{array}{l}\text { Cardiovascular death, } \\
\text { aborted cardiac arrest or } \\
\text { first hospitalization for HF }\end{array}$ & 40 & 57 \\
\hline J-DHF & 2014 & 245 & Carvedilol & $\beta$-Blocker & $>40$ & HFpEF & $\begin{array}{l}\text { Cardiovascular death or } \\
\text { first hospitalization for HF }\end{array}$ & 39 & 169 \\
\hline PARAGON-HF & 2019 & 4,822 & $\begin{array}{l}\text { Sacubitril- } \\
\text { valsartan }\end{array}$ & ARNI & $\geq 45$ & HFpEF & $\begin{array}{l}\text { Cardiovascular death } \\
\text { or total hospitalizations } \\
\text { for HF }\end{array}$ & 35 & 59 \\
\hline SPIRIT-HF & $\begin{array}{l}\text { 2017- } \\
\text { ongoing }\end{array}$ & 1,300 & Spironolactone & MRA & $\geq 40$ & HFpEF & $\begin{array}{l}\text { Cardiovascular death } \\
\text { or total hospitalizations } \\
\text { for HF }\end{array}$ & Ongoing & 54 \\
\hline EMPEROR-Preserved & $\begin{array}{l}\text { 2017- } \\
\text { ongoing }\end{array}$ & 5,988 & Empagliflozin & SGLT2i & $>40$ & HFpEF & $\begin{array}{l}\text { Cardiovascular death or } \\
\text { first hospitalization for HF }\end{array}$ & Ongoing & 148 \\
\hline EMPERIALPreserved & $\begin{array}{l}\text { 2018- } \\
\text { ongoing }\end{array}$ & 315 & Empagliflozin & SGLT2i & $>40$ & HFpEF & $\begin{array}{l}\text { Changes in 6-min walking } \\
\text { distance }\end{array}$ & Ongoing & 149 \\
\hline DELIVER & $\begin{array}{l}\text { 2018- } \\
\text { ongoing }\end{array}$ & 6,100 & Dapagliflozin & SGLT2i & $>40$ & HFpEF & $\begin{array}{l}\text { Cardiovascular death, } \\
\text { first hospitalization for } \\
\text { HF or urgent hospital visit } \\
\text { for HF }\end{array}$ & Ongoing & 53 \\
\hline DETERMINE-PRESERVED & $\begin{array}{l}\text { 2019- } \\
\text { ongoing }\end{array}$ & 504 & Dapagliflozin & SGLT2i & $>40$ & HFpEF & KCCQ-TSS changes & Ongoing & 150 \\
\hline PARAGLIDE-HF & $\begin{array}{l}\text { 2019- } \\
\text { ongoing }\end{array}$ & 800 & $\begin{array}{l}\text { Sacubitril- } \\
\text { valsartan }\end{array}$ & ARNI & $>40$ & HFpEF & NT-proBNP changes & Ongoing & 170 \\
\hline FINEARTS-HF & $\begin{array}{l}2020- \\
\text { ongoing }\end{array}$ & 5,500 & Finerenone & MRA & $\geq 40$ & HFpEF & $\begin{array}{l}\text { Cardiovascular death } \\
\text { or total hospitalizations } \\
\text { for HF }\end{array}$ & Ongoing & 171 \\
\hline
\end{tabular}

Treatment effects for selected trials are shown in FIG. 6. ACEi, angiotensin-converting enzyme inhibitor; ARB, angiotensin-receptor blocker; ARNI, angiotensinreceptor blocker-neprilysin inhibitor; CRT, cardiac resynchronization therapy; EF, ejection fraction; HF, heart failure; HFmrEF, heart failure with mildly reduced ejection fraction; HFpEF, heart failure with preserved ejection fraction; HFrEF, heart failure with reduced ejection fraction; KCCQ-TSS, Kansas City Cardiomyopathy Questionnaire total symptoms score; LVWMI, left ventricular wall motion index; MI, myocardial infarction; MRA, mineralocorticoid-receptor antagonist; NT-proBNP, $\mathrm{N}$-terminal pro-B-type natriuretic peptide; sGC, soluble guanylate cyclase; SGLT2i, sodium-glucose cotransporter 2 inhibitor. 


\begin{tabular}{|c|c|c|c|c|}
\hline Study & Outcome & $\mathrm{EF}(\%)$ & HFrEF $\square$ HFmrEF $\square \mathrm{HFpEF}$ & $\mathrm{HR}(95 \% \mathrm{Cl})$ \\
\hline \multirow[t]{2}{*}{$\begin{array}{l}\text { CHARM } \\
\text { programme }\end{array}$} & $\begin{array}{l}\mathrm{CV} \text { death } \\
\text { or HHF }\end{array}$ & $\begin{array}{l}<40 \\
40-49 \\
\geq 50\end{array}$ & 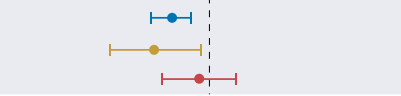 & $\begin{array}{l}0.82(0.75-0.91) \\
0.76(0.61-0.96) \\
0.95(0.79-1.14)\end{array}$ \\
\hline & $\begin{array}{l}\text { Recurrent } \\
\mathrm{HHF}\end{array}$ & $\begin{array}{l}<40 \\
40-49 \\
\geq 50\end{array}$ & $\stackrel{\longmapsto}{\longmapsto}$ & $\begin{array}{l}0.68(0.58-0.80) \\
0.48(0.33-0.70) \\
0.78(0.59-1.03)\end{array}$ \\
\hline PEP-CHF & $\begin{array}{l}\text { All-cause death } \\
\text { or HHF }\end{array}$ & $40-49$ & $\longmapsto:-1$ & $0.92(0.70-1.21)$ \\
\hline \multirow[t]{2}{*}{$\begin{array}{l}\text { DIG } \\
\text { (ancillary) }\end{array}$} & $\begin{array}{l}\mathrm{CV} \text { death } \\
\text { or HHF }\end{array}$ & $\begin{array}{l}<40 \\
40-49 \\
\geq 50\end{array}$ & 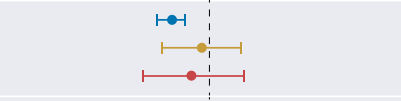 & $\begin{array}{l}0.83(0.77-0.89) \\
0.96(0.79-1.17) \\
0.92(0.71-1.20)\end{array}$ \\
\hline & $\begin{array}{l}\text { HF death } \\
\text { or HHF }\end{array}$ & $\begin{array}{l}<40 \\
40-49 \\
\geq 50\end{array}$ & $\begin{array}{l:l}\longmapsto-1 & 1 \\
\longmapsto & \longmapsto\end{array}$ & $\begin{array}{l}0.74(0.68-0.81) \\
0.83(0.66-1.05) \\
0.88(0.65-1.19)\end{array}$ \\
\hline \multirow[t]{2}{*}{ PEACE } & CV death & $40-49$ & $\longmapsto: \begin{array}{ccc}1 \\
1\end{array}$ & $0.88(0.72-1.09)$ \\
\hline & $\mathrm{HHF}$ & $40-49$ & \begin{tabular}{lll}
$1 \longmapsto$ \\
\hdashline$\vdots 1$
\end{tabular} & $0.96(0.78-1.19)$ \\
\hline \multirow[t]{2}{*}{ TOPCAT } & $\begin{array}{l}\text { CV death, } \\
\text { CA or HHF }\end{array}$ & $\begin{array}{l}<50 \\
50-54.99 \\
55-59.99 \\
\geq 60\end{array}$ & \begin{tabular}{l:ll}
$\longmapsto$ \\
\hdashline
\end{tabular} & $\begin{array}{l}0.72(0.50-1.05) \\
0.85(0.61-1.18) \\
0.94(0.68-1.29) \\
0.97(0.76-1.23)\end{array}$ \\
\hline & $\mathrm{HHF}$ & $\begin{array}{l}<50 \\
50-54.99 \\
55-59.99 \\
\geq 60\end{array}$ & 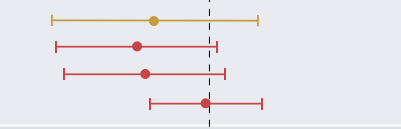 & $\begin{array}{l}0.76(0.46-1.27) \\
0.70(0.47-1.04) \\
0.73(0.49-1.08) \\
0.98(0.74-1.30)\end{array}$ \\
\hline \multirow[t]{2}{*}{$\begin{array}{l}\text { TOPCAT } \\
\text { Americas }\end{array}$} & $\begin{array}{l}\text { CV death, } \\
\text { CA or HHF }\end{array}$ & $\begin{array}{l}<50 \\
50-54.99 \\
55-59.99 \\
\geq 60\end{array}$ & $\begin{array}{ll:l}\longmapsto \longmapsto \\
\longmapsto \longmapsto\end{array}$ & $\begin{array}{l}0.55(0.33-0.91) \\
0.83(0.56-1.25) \\
0.85(0.60-1.21) \\
0.89(0.69-1.15)\end{array}$ \\
\hline & $\mathrm{HHF}$ & $\begin{array}{l}<50 \\
50-54.99 \\
55-59.99 \\
\geq 60\end{array}$ & $\begin{array}{l:l}\longmapsto \\
\longmapsto\end{array}$ & $\begin{array}{l}0.60(0.32-1.10) \\
0.80(0.51-1.25) \\
0.70(0.47-1.06) \\
0.95(0.71-1.26)\end{array}$ \\
\hline \multirow[t]{2}{*}{ BB-meta-HF } & CV death & $\begin{array}{l}<40 \\
40-49 \\
\geq 50\end{array}$ & 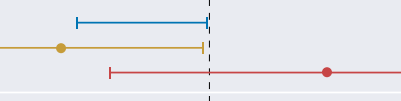 & $\begin{array}{l}0.67-0.78(0.52-0.65 \text { to } 0.80-0.99) \\
0.48(0.24-0.97) \\
1.77(0.61-5.14)\end{array}$ \\
\hline & $\begin{array}{l}\mathrm{CV} \text { death } \\
\text { or } \mathrm{CVH}\end{array}$ & $\begin{array}{l}<40 \\
40-49 \\
\geq 50\end{array}$ & \begin{tabular}{l:l}
$\longmapsto$ & \\
\hdashline & $\longmapsto$ \\
\hdashline
\end{tabular} & $\begin{array}{l}0.68-0.80(0.60-0.72 \text { to } 0.77-0.88) \\
0.83(0.60-1.13) \\
0.66(0.38-1.15)\end{array}$ \\
\hline \multirow[t]{3}{*}{$\begin{array}{l}\text { PARADIGM-HF- } \\
\text { PARAGON-HF }\end{array}$} & $\begin{array}{l}\mathrm{CV} \text { death } \\
\text { or } \mathrm{HHF}\end{array}$ & $\begin{array}{l}\leq 42.5 \\
42.5-52.5 \\
\geq 52.5\end{array}$ & 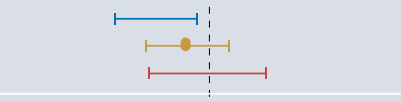 & $\begin{array}{l}0.77-0.81(0.63-0.71 \text { to } 0.92-0.94) \\
0.89(0.73-1.10) \\
0.89-1.03(0.74-0.80 \text { to } 1.06-1.32)\end{array}$ \\
\hline & Total HHF & $\begin{array}{l}\leq 42.5 \\
42.5-52.5 \\
\geq 52.5\end{array}$ & \begin{tabular}{l:c} 
\\
\hdashline
\end{tabular} & $\begin{array}{l}0.75-0.82(0.60-0.63 \text { to } 0.90-1.11) \\
0.77(0.58-1.02) \\
0.81-1.04(0.63-0.76 \text { to } 1.04-1.44)\end{array}$ \\
\hline & & & $\begin{array}{c}1.000 \\
\operatorname{HR}(95 \% \mathrm{Cl})\end{array}$ & \\
\hline
\end{tabular}

Fig. 6 | Outcomes in patients with HF according to EF. Forest plot depicting hazard ratio (HR) and $95 \%$ confidence interval $(\mathrm{Cl})$ for intervention versus control according to left ventricular ejection fraction $(E F)$ in patients with heart failure (HF) with reduced $E F(H F r E F), H F$ with mildly reduced $E F$ (HFmrEF) and $H F$ with preserved $E F(H F p E F)$ for the specified trials and outcomes. In the CHARM programme ${ }^{51,72}$, therapy with candesartan reduced the risk of cardiovascular (CV) death and hospitalization for HF (HHF) in patients with HFrEF or HFmrEF but not in those with HFpEF. In PEP-CHF ${ }^{146}$, perindopril therapy was not effective in reducing the risk of all-cause death or HHF in elderly patients with mildly impaired systolic dysfunction. A retrospective analysis of the DIG trial ${ }^{167}$ did not detect a benefit of digoxin therapy when the EF was $>40 \%$. The PEACE trial ${ }^{156,166}$ did not show a reduction in the risk of $\mathrm{CV}$ death and $\mathrm{HHF}$ with angiotensin-converting enzyme inhibitors in patients with ischaemic $\mathrm{HF}$ with mildly reduced EF. In the TOPCAT Americas substudy ${ }^{172}$, the risk of the primary composite end point of $\mathrm{CV}$ death, HHF or aborted cardiac arrest (CA) was reduced with spironolactone therapy in patients with $E F$ at the lower end of the included $E F$ spectrum ( $E F \geq 45 \%)$. The BB-meta-HF study ${ }^{99}$, a large meta-analysis of trials on $\beta$-blockers, demonstrated a lower all-cause and $\mathrm{CV}$ mortality in patients in sinus rhythm with HFrEF or HFmrEF but not in those with HFpEF. In a prespecified subgroup analysis of the PARAGON-HF trial ${ }^{59}$, therapy with sacubitril-valsartan was effective in patients with EF lower than or the same as the median (EF 57\%). For the $\mathrm{HFrEF}(\mathrm{EF}<40 \%$ ) category of the BB-meta-HF study, we report the ranges of $\mathrm{HR}$ and $95 \% \mathrm{Cl}$ across the subgroups of $\mathrm{HFrEF}$ considered in the study (EF $<20 \%, 20-25 \%, 26-34 \%$ and 35-39\%). For the HFrEF and HFpEF categories of the PARADIGM-HF-PARAGON-HF study, we report the ranges of $\mathrm{HR}$ and $95 \% \mathrm{Cl}$ across the subgroups of $\mathrm{HFrEF}$ ( $\mathrm{EF} \leq 22.5 \%$, $22.5-32.5 \%$ and $>32.5-42.5 \%$ ) and HFpEF (EF > 52.5-62.5\% and >62.5\%) considered in the study. category, when repeat events rather than time to first event were analysed, candesartan significantly reduced the risk of cardiovascular death or recurrent hospitalization for HF by $25 \%{ }^{155}$. In a post hoc analysis of the PEACE trial $^{156}$, which enrolled patients with stable coronary artery disease and normal or slightly reduced EF, the angiotensin-converting enzyme inhibitor trandolapril improved survival and a composite of death, myocardial infarction and stroke compared with placebo in patients with HFmrEF but did not reduce cardiovascular 
mortality and hospitalizations for HF. In TOPCAT ${ }^{87}$, which enrolled patients with $\mathrm{HF}$ with $\mathrm{EF} \geq 45 \%$, a significant interaction between treatment and $\mathrm{EF}$ and outcomes was observed for both the primary end point (a composite of cardiovascular death, hospitalization for $\mathrm{HF}$ and aborted cardiac arrest) and the secondary end point of hospitalization for HF. Spline analyses highlighted a significant treatment effect with spironolactone in terms of reduced risk of the primary outcome or hospitalization for HF when the EF moved below $55 \%{ }^{87}$. Additionally, spironolactone significantly reduced the primary outcome when data from North and South America were separately analysed, as well as in the subgroup of patients who were included in the trial based on their high natriuretic peptide levels, which would increase the likelihood of the true presence of $\mathrm{HF}^{87,157}$. Accordingly, in December 2020, the FDA advisory committee recommended with an $8 / 4$ vote that existing evidence supported the use of spironolactone for the reduction of hospitalizations for HF in patients with HFpEF, specifying that the benefit might be expected in patients in the mildly reduced range of $\mathrm{EF}$ (with comments made about EF of $45-55 \%$ or up to $57 \%)^{158}$.

An individual patient-data meta-analysis of 11 RCTs of $\beta$-blockers demonstrated the benefit of this therapy for the reduction of all-cause and cardiovascular mortality in patients in sinus rhythm with HFrEF or HFmrEF but not in those with $\mathrm{HFpEF}^{99}$. In a retrospective analysis of the DIG trial ${ }^{159}$, therapy with digoxin significantly reduced cardiovascular death or hospitalizations for HF in patients with HFrEF but not in patients with HFpEF or HFmrEF. This finding was confirmed in a spline analysis in which the digoxin treatment effect became nonsignificant when the EF changed above $40 \%{ }^{159}$. In 2020, in the PARAGON-HF trial involving patients with $\mathrm{HF}$ with $\mathrm{EF} \geq 45 \%$, treatment with sacubitrilvalsartan was effective in patients with $\mathrm{EF}$ equal to or below the median $(\mathrm{EF} \leq 57 \%)$, which was also shown when the relationship between continuous EF values

\section{Box 2 | Knowledge gaps and future directions}

- The advancing concept of precision medicine is based on deep phenotyping and individualization of treatments and might replace or complement the current left ventricular ejection fraction (EF)-based approach to diagnosis and categorization of heart failure. Integrating emerging imaging techniques, advanced analytics, multiple biomarkers and omics with $\mathrm{EF}$ assessment might provide more precise phenotyping and targeted treatment.

- Heart failure with mildly reduced EF (HFmrEF) is a heterogeneous category that is based on a snapshot EF measurement in the dynamic natural history that characterizes the heart failure syndrome. Current data provide an extensive but static characterization of HFmrEF. Therefore, there is a need for a comprehensive assessment of $\mathrm{EF}$ trajectories over time and their interaction with multiple patient characteristics that also change over time in complex, time-dependent models.

- Strategies for the prevention of sudden cardiac death in patients with $E F \geq 40 \%$ have not been tested. The potential role of device-based therapies, such as cardiac resynchronization therapy, is unknown.

- Future treatment of HFmrEF might rely on weak guideline recommendations that are based on post hoc and subgroup analyses of completed trials of heart failure that have included patients with HFmrEF. However, trials evaluating existing therapies for heart failure with reduced EF will also need to be assessed in dedicated HFmrEF trials, that is, trials on the new use of existing drugs or 'repurposing' trials. These studies are less likely to be sponsored by industry and are suitable for publicly funded, pragmatic trials. and treatment effect was analysed by spline analyses ${ }^{26,59}$. An expanded indication for sacubitril-valsartan was granted in 2020 by the FDA, with the clarification that the most benefit is expected in patients with EF below normal values ${ }^{160}$. Altogether, these data suggest that the use of angiotensin-converting enzyme inhibitors, angiotensin-receptor blockers, $\beta$-blockers, MRAs and sacubitril-valsartan can be considered in HFmrEF ${ }^{161}$. The ongoing, registry-based RCT SPIRRIT-HFpEF ${ }^{53}$ enrolling patients with $\mathrm{EF} \geq 40 \%$ (HFmrEF and $\mathrm{HFpEF}$ ) will provide further important evidence regarding the potential benefit of MRAs in the entire HFmrEF and HFpEF spectrum.

Novel drugs that act on alternative pathways are emerging for the treatment of HF and have been demonstrated to be effective in the setting of HFrEF in the past few years. Three large RCTs have tested treatment with SGLT2 inhibitors in patients with HF. The risk of cardiovascular death or hospitalization for HF was significantly reduced by empagliflozin in the EMPEROR-Reduced trial $^{36}$ and by dapagliflozin in the DAPA-HF trial ${ }^{35}$ (which enrolled patients with HFrEF with or without diabetes). These results have generated great expectations for the potential of SGLT2 inhibitors for the treatment of HFmrEF and HFpEF, which is currently being tested in the EMPEROR-Preserved and DELIVER trials $^{162}$. The first data supporting the use of SGLT2 inhibitors in patients with HFmrEF or HFpEF come from the SOLOIST-WHF trial ${ }^{17}$. Patients with HF and type 2 diabetes who were stabilized after hospitalization for worsening HF or recently discharged from hospital were randomly assigned, regardless of EF, to the SGLT2-SGLT1 inhibitor sotagliflozin or placebo. The benefits of sotagliflozin for risk reduction of cardiovascular death or hospitalizations or urgent visits for $\mathrm{HF}$ was consistent in patients with $\mathrm{EF}<50 \%$ or $\geq 50 \%{ }^{17}$.

A proportion of patients with HFmrEF are those in whom the EF has improved from HFrEF owing to the successful use of HFrEF therapies ${ }^{122}$. In this setting, available data support the indefinite continuation of HFrEF treatments, and the withdrawal of neurohormonal antagonists after the improvement (or recovery) of EF should be strongly discouraged ${ }^{138}$. In the TRED-HF trial $^{154}$, HFrEF treatments were withdrawn in a small group of patients with dilated cardiomyopathy and recovered EF. After weaning, about $40 \%$ of patients experienced a recurrence of $\mathrm{HF}$ within 6 months, defined by a fall in $\mathrm{EF}$ of $>10 \%$ to $<50 \%$, an increase in left ventricular end-diastolic volume of $>10 \%$ to greater than the normal range, a doubling of the NT-proBNP level to $>400 \mathrm{ng} / \mathrm{l}$, or clinical evidence of HF, but no deaths were observed in this small, short-term trial ${ }^{154}$. However, whether patients with recovered EF who have not yet received HFrEF therapy should be started on this therapy after EF has already recovered remains unknown.

The use of device therapy in patients with HFmrEF has no supporting data. However, a growing subgroup of patients who receive an ICD for primary prevention have an improvement in EF to HFmrEF or HFpEF at the time of generator replacement and, therefore, no longer fulfil the criteria for an ICD for primary prevention ${ }^{6}$. In a retrospective study, albeit lower than in patients with 
persisting ICD indication, patients who did not fulfil the criteria for an ICD had an annual rate of appropriate ICD interventions of around $3 \%{ }^{163}$. A retrospective analysis of the SCD-HeFT trial ${ }^{164}$ investigated patients with available reassessment of EF at a median interval of 13 months after ICD implantation and showed that the subsequent benefit in reduced mortality that was gained by receiving an ICD was similar in patients who improved to EF $>35 \%$ and those with persisting EF $\leq 35 \%$. Therefore, the available evidence suggests that the risk of arrhythmia persists at least to some extent in patients with improved or recovered $\mathrm{EF}$.

\section{Conclusions}

After the introduction of the mid-range EF category of HF in the 2016 ESC HF guidelines ${ }^{6}$, many studies have contributed to improving our understanding of the epidemiology, clinical characteristics, prognosis and potential treatment effects in patients with HFmrEF.
The overall picture supports the notion that HFmrEF is more similar to HFrEF than to HFpEF, especially in aetiology and treatment response, and therefore is more appropriately termed HF with mildly reduced $\mathrm{EF}^{19,20}$. Gaps in our knowledge need to be filled, and future dedicated research should encompass the whole scenario of HFmrEF, from pathogenesis to treatment (BOX 2).

A broad range of effective therapies for HFrEF is now available. HFpEF seems to be distinctly different from HFrEF and is likely to need more targeted treatments and dedicated clinical trials. Patients with HFmrEF seem to be responsive to HFrEF medications but the strength of recommendations and level of evidence for these drugs in patients with HFmrEF must be considered modest. Ongoing clinical trials in patients with $\mathrm{HFmrEF}$ or HFpEF, in particular of MRAs and SGLT2 inhibitors, will inform the future treatment landscape in HFmrEF.

\section{Published online 6 September 2021}

1. Conrad, N. et al. Temporal trends and patterns in heart failure incidence: a population-based study of 4 million individuals. Lancet 391, 572-580 (2018).

2. Jernberg, T. et al. Association between adoption of evidence-based treatment and survival for patients with ST-elevation myocardial infarction. JAMA 305, 1677-1684 (2011)

3. van Riet, E. E. et al. Epidemiology of heart failure the prevalence of heart failure and ventricular dysfunction in older adults over time. A systematic review. Eur. J. Heart Fail. 18, 242-252 (2016).

4. Taylor, C. J. et al. Trends in survival after a diagnosis of heart failure in the United Kingdom 2000-2017: population based cohort study. BMJ 364, 1223 (2019)

5. Yancy, C. W. et al. 2017 ACC/AHA/HFSA focused update of the 2013 ACCF/AHA Guideline for the Management of Heart Failure: a report of the American College of Cardiology/American Heart Association Task Force on Clinical Practice Guidelines and the Heart Failure Society of America. Circulation 136, e137-e161 (2017).

6. Ponikowski, P. et al. 2016 ESC Guidelines for the diagnosis and treatment of acute and chronic heart failure: the Task Force for the diagnosis and treatment of acute and chronic heart failure of the European Society of Cardiology (ESC) developed with the special contribution of the Heart Failure Association (HFA) of the ESC. Eur. Heart J. 37, 2129-2200 (2016).

7. Virani, S. S. et al. Heart disease and stroke statistics - 2021 update: a report from the American Heart Association. Circulation 143, e254-e743 (2021).

8. Thorvaldsen, T., Benson, L., Dahlstrom, U., Edner, M. \& Lund, L. H. Use of evidence-based therapy and survival in heart failure in Sweden 2003-2012. Eur. J. Heart Fail. 18, 503-511 (2016).

9. Ambrosy, A. P. et al. The global health and economic burden of hospitalizations for heart failure: lessons learned from hospitalized heart failure registries. J. Am. Coll. Cardiol. 63, 1123-1133 (2014).

10. Lund, L. H., Benson, L., Dahlstrom, U. \& Edner, M. Association between use of renin-angiotensin system antagonists and mortality in patients with heart failure and preserved ejection fraction. JAMA 308 , 2108-2117 (2012).

11. Lund, L. H. et al. Association between enrolment in a heart failure quality registry and subsequent mortality - a nationwide cohort study. Eur. J. Heart Fail. 19,1107-1116 (2017).

12. Crespo-Leiro, M. G. et al. Advanced heart failure: a position statement of the Heart Failure Association of the European Society of Cardiology. Eur. J. Heart Fail. 20, 1505-1535 (2018).

13. Ahmad, T. et al. Machine learning methods improve prognostication, identify clinically distinct phenotypes and detect heterogeneity in response to therapy in a large cohort of heart failure patients. J. Am. Heart Assoc. 7, e008081 (2018)

14. Lund, L. H. Heart failure with "mid-range" ejection fraction - new opportunities. J. Card. Fail. 22, 769-771 (2016).
15. Lund, L. H. Vedin, O \& Savarese, G. Is ejection fraction in heart failure a limitation or an opportunity? Eur. J. Heart Fail. 20, 431-432 (2018).

16. Marwick, T. H. Ejection fraction pros and cons: JACC state-of-the-art review. J. Am. Coll. Cardiol. 72, 2360-2379 (2018)

17. Bhatt, D. L. et al. Sotagliflozin in patients with diabetes and recent worsening heart Failure. N. Engl. J. Med. 384, 117-128 (2020).

18. Lam, C. S. \& Solomon, S. D. The middle child in heart failure: heart failure with mid-range ejection fraction (40-50\%). Eur. J. Heart Fail. 16, 1049-1055 (2014).

19. Bozkurt, B. et al. Universal definition and classification of heart failure: a report of the Heart Failure Society of America, Heart Failure Association of the European Society of Cardiology, Japanese Heart Failure Society and Writing Committee of the Universal Definition of Heart Failure: Endorsed by Canadian Heart Failure Society, Heart Failure Association of India, the Cardiac Society of Australia and New Zealand, and the Chinese Heart Failure Association. Eur. J. Heart Fail. 23, 352-380 (2021)

20. Lam, C. S. P., Voors, A. A., Piotr, P., McMurray, J. J. V. $\&$ Solomon, S. D. Time to rename the middle child of heart failure: heart failure with mildly reduced ejection fraction. Eur. Heart J. 41, 2353-2355 (2020).

21. Kou, S. et al. Echocardiographic reference ranges for normal cardiac chamber size: results from the NORRE study. Eur. Heart J. Cardiovasc. Imaging 15, 680-690 (2014)

22. Borlaug, B. A. \& Redfield, M. M. Diastolic and systolic heart failure are distinct phenotypes within the heart failure spectrum. Circulation 123, 2006-2013; discussion 2014 (2011).

23. Dunlay, S. M., Roger, V. L., Weston, S. A., Jiang, R. $\&$ Redfield, M. M. Longitudinal changes in ejection fraction in heart failure patients with preserved and reduced ejection fraction. Circ. Heart Fail. 5 , 720-726 (2012)

24. Lang, R. M. et al. Recommendations for cardiac chamber quantification by echocardiography in adults: an update from the American Society of Echocardiography and the European Association of Cardiovascular Imaging. Eur. Heart J. Cardiovasc. Imaging 16, 233-270 (2015).

25. Galderisi, M. et al. Standardization of adult transthoracic echocardiography reporting in agreemen with recent chamber quantification, diastolic function, and heart valve disease recommendations: an expert consensus document of the European Association of Cardiovascular Imaging. Eur. Heart J. Cardiovasc. Imaging 18, 1301-1310 (2017).

26. Solomon, S. D. et al. Sacubitril/valsartan across the spectrum of ejection fraction in heart failure. Circulation 141, 352-361 (2020).

27. Dougherty, A. H., Naccarelli, G. V., Gray, E. L., Hicks, C. H. \& Goldstein, R. A. Congestive heart failure with normal systolic function. Am. J. Cardiol. 54, 778-782 (1984)
28. Soufer $\mathrm{R}$ et al. Intact systolic left ventricular function in clinical congestive heart failure. Am. J. Cardiol. 55 1032-1036 (1985).

29. Armstrong, P. W. et al. Vericiguat in patients with heart failure and reduced ejection fraction. N. Engl. J. Med. 382, 1883-1893 (2020).

30. Cohn, J. N. \& Tognoni, G., Valsartan Heart Failure Trial Investigators. A randomized trial of the angiotensin-receptor blocker valsartan in chronic heart failure. N. Engl. J. Med. 345, 1667-1675 (2001).

31. No Authors Listed. Effect of metoprolol $\mathrm{CR} / \mathrm{XL}$ in chronic heart failure: metoprolol CR/XL randomised intervention trial in congestive heart failure (MERIT-HF). Lancet 353, 2001-2007 (1999).

32. Young, J. B. et al. Mortality and morbidity reduction with candesartan in patients with chronic heart failure and left ventricular systolic dysfunction: results of the CHARM low-left ventricular ejection fraction trials. Circulation 110, 2618-2626 (2004).

33. No Authors Listed. A randomized trial of betablockade in heart failure. The Cardiac Insufficiency Bisoprolol Study (CIBIS). CIBIS Investigators and Committees. Circulation 90, 1765-1773 (1994).

34. McMurray, J. J. et al. Angiotensin-neprilysin inhibition versus enalapril in heart failure. N. Engl. J. Med. 371, 993-1004 (2014).

35. McMurray, J. J. V. et al. Dapagliflozin in patients with heart failure and reduced ejection fraction. N. Engl. J. Med. 381, 1995-2008 (2019).

36. Packer, M. et al. Cardiovascular and renal outcomes with empagliflozin in heart failure. N. Engl. J. Med. 383, 1413-1424 (2020).

37. Zannad, F. et al. Eplerenone in patients with systolic heart failure and mild symptoms. N. Engl. J. Med. 364, 11-21 (2011).

38. Pitt, B. et al. The effect of spironolactone on morbidity and mortality in patients with severe heart failure. Randomized aldactone evaluation study investigators N. Engl. J. Med. 341, 709-717 (1999).

39. Investigators, $\mathrm{S}$, et al. Effect of enalapril on survival in patients with reduced left ventricular ejection fractions and congestive heart failure. N. Engl. J. Med. 325 , 293-302 (1991).

40. Bardy, G. H. et al. Amiodarone or an implantable cardioverter-defibrillator for congestive heart failure. N. Engl. J. Med. 352, 225-237 (2005).

41. Bristow, M. R. et al. Cardiac-resynchronization therapy with or without an implantable defibrillator in advanced chronic heart failure. N. Engl. J. Med. 350, 2140-2150 (2004).

42. Teerlink, J. R. et al. Cardiac myosin activation with omecamtiv mecarbil in systolic heart failure. N. Engl. J. Med. (2020).

43. Moss, A. J. et al. Prophylactic implantation of a defibrillator in patients with myocardial infarction and reduced ejection fraction. N. Engl. J. Med. 346 877-883 (2002).

44. Moss, A. J. et al. Cardiac-resynchronization therapy for the prevention of heart-failure events. N. Engl. J. Med. 361, 1329-1338 (2009). 
45. Packer, M. et al. Effect of carvedilol on survival in severe chronic heart failure. N. Engl. J. Med. 344 1651-1658 (2001)

46. Savarese, G. et al. Utilizing NT-proBNP for eligibility and enrichment in trials in HFpEF, HFmrEF, and HFrEF. JACC Heart Fail. 6, 246-256 (2018).

47. Meta-analysis Global Group in Chronic Heart Failure. The survival of patients with heart failure with preserved or reduced left ventricular ejection fraction: an individual patient data meta-analysis. Eur. Heart $J$. 33, 1750-1757 (2012)

48. Koh, A. S. et al. A comprehensive population-based characterization of heart failure with mid-range ejection fraction. Eur. J. Heart Fail. 19, 1624-1634 (2017).

49. Wehner, G. J. et al. Routinely reported ejection fraction and mortality in clinical practice: where does the nadir of risk lie? Eur. Heart J. 41, 1249-1257 (2020).

50. Toma, M. et al. The relationship between left ventricular ejection fraction and mortality in patients with acute heart failure: insights from the ASCEND-HF Trial. Eur. J. Heart Fail. 16, 334-341 (2014).

51. Yusuf, S. et al. Effects of candesartan in patients with chronic heart failure and preserved left-ventricular ejection fraction: the CHARM-Preserved Trial Lancet 362, 777-781 (2003).

52. Anker, S. D. et al. Evaluation of the effects of sodium-glucose co-transporter 2 inhibition with empagliflozin on morbidity and mortality in patients with chronic heart failure and a preserved ejection fraction: rationale for and design of the EMPEROR-Preserved trial. Eur. J. Heart Fail. 21, 1279-1287 (2019).

53. US National Library of Medicine. ClinicalTrials.gov http://clinicaltrials.gov/ct2/show/NCT03619213 (2021)

54. EU Clinical Trials Register https://www clinicaltrialsregister.eu/ctr-search/search? query $=2017-000697-11$ (2021)

55. US National Library of Medicine. ClinicalTrials.gov https://clinicaltrials.gov/ct2/show/NCT02901184 (2021).

56. Lund, L. H., Oldgren, J. \& James, S. Registry-based pragmatic trials in heart failure: current experience and future directions. Curr. Heart Fail. Rep. 14, 59-70 (2017).

57. Pitt, B. et al. Spironolactone for heart failure with preserved ejection fraction. N. Engl. J. Med. 370 1383-1392 (2014)

58. Massie, B. M. et al. Irbesartan in patients with heart failure and preserved ejection fraction. N. Engl. J. Med. 359, 2456-2467 (2008).

59. Solomon, S. D. et al. Angiotensin-neprilysin inhibition in heart failure with preserved ejection fraction. N. Engl. J. Med. 381, 1609-1620 (2019).

60. Bhatt, D. L. et al. Sotagliflozin in patients with diabetes and recent worsening heart failure. N. Engl. J. Med. 384, 117-128 (2021).

61. Yancy, C. W. et al. 2013 ACCF/AHA guideline for the management of heart failure: executive summary: a report of the American College of Cardiology Foundation/American Heart Association Task Force on practice guidelines. Circulation 128, 1810-1852 (2013).

62. Savarese, G. \& Lund, L. H. Global public health burden of heart failure. Card. Fail. Rev. 3, 7-11 (2017).

63. Groenewegen, A., Rutten, F. H., Mosterd, A. \& Hoes, A. W. Epidemiology of heart failure. Eur. J. Heart Fail. 22, 1342-1356 (2020).

64. Bhambhani, V. et al. Predictors and outcomes of heart failure with mid-range ejection fraction. Eur. J. Heart Fail. 20, 651-659 (2018).

65. Chioncel, O. et al. Epidemiology and one-year outcomes in patients with chronic heart failure and preserved, mid-range and reduced ejection fraction: an analysis of the ESC heart failure long-term registry. Eur. J. Heart Fail. 19, 1574-1585 (2017).

66. Douglas, P. S. et al. Echocardiographic imaging in clinical trials: American Society of Echocardiography Standards for echocardiography core laboratories: endorsed by the American College of Cardiology Foundation. J. Am. Soc. Echocardiogr. 22, 755-765 (2009).

67. Kapoor, J. R. et al. Precipitating clinical factors, heart failure characterization, and outcomes in patients hospitalized with heart failure with reduced, borderline, and preserved ejection fraction JACC Heart Fail. 4, 464-472 (2016).
68. Lam, C. S. P. et al. Mortality associated with heart failure with preserved vs. reduced ejection fraction in a prospective international multi-ethnic cohort study. Eur. Heart J. 39, 1770-1780 (2018).

69. Rickenbacher, P. et al. Heart failure with mid-range ejection fraction: a distinct clinical entity? Insights from the Trial of Intensified versus standard Medical therapy in Elderly patients with Congestive Heart Failure (TIME-CHF). Eur. J. Heart Fail. 19 , 1586-1596 (2017).

70. Tsuji, K. et al. Characterization of heart failure patients with mid-range left ventricular ejection fraction-a report from the CHART-2 Study. Eur. J. Heart Fail. 19 1258-1269 (2017).

71. Steinberg, B. A. et al. Trends in patients hospitalized with heart failure and preserved left ventricular ejection fraction: prevalence, therapies, and outcomes. Circulation 126, 65-75 (2012).

72. Lund, L. H. et al. Heart failure with mid-range ejection fraction in CHARM: characteristics, outcomes and effect of candesartan across the entire ejection fraction spectrum. Eur. J. Heart Fail. 20, 1230-1239 (2018).

73. Srivastava, P. K., Hsu, J. J., Ziaeian, B. \& Fonarow, G. C. Heart failure with mid-range ejection fraction. Curr. Heart Fail. Rep. 17, 1-8 (2020).

74. Fonarow, G. C. et al. Characteristics, treatments, and outcomes of patients with preserved systolic function hospitalized for heart failure: a report from the OPTIMIZE-HF registry. J. Am. Coll. Cardiol. 50 768-777 (2007)

75. Shah, K. S. et al. Heart failure with preserved, borderline, and reduced ejection fraction: 5-year outcomes. J. Am. Coll. Cardiol. 70, 2476-2486 (2017).

76. Sweitzer, N. K., Lopatin, M., Yancy, C. W., Mills, R. M. \& Stevenson, L. W. Comparison of clinical features and outcomes of patients hospitalized with heart failure and normal ejection fraction ( $>$ or $=55 \%$ ) versus those with mildly reduced $(40 \%$ to $55 \%)$ and moderately to severely reduced $(<40 \%)$ fractions. Am. J. Cardiol. 101, 1151-1156 (2008).

77. Kalogeropoulos, A. P. et al. Characteristics and outcomes of adult outpatients with heart failure and improved or recovered ejection fraction. JAMA Cardiol. 1, 510-518 (2016)

78. Vedin, $\mathrm{O}$. et al. Significance of ischemic heart disease in patients with heart failure and preserved, midrange, and reduced ejection fraction: a nationwide cohort study. Circ Heart Fail. 10, e003875 (2017).

79. Stolfo, D. et al. Sex-based differences in heart failure across the ejection fraction spectrum: phenotyping, and prognostic and therapeutic implications. JACC Heart Fail. 7, 505-515 (2019).

80. Savarese, G. et al. Associations with and prognostic and discriminatory role of $\mathrm{N}$-terminal pro-b-type natriuretic peptide in heart failure with preserved versus mid-range versus reduced ejection fraction. J. Card. Fail. 24, 365-374 (2018).

81. Tromp, J. et al. Biomarker profiles of acute heart failure patients with a mid-range ejection fraction. JACC Heart Fail. 5, 507-517 (2017).

82. Sartipy, U., Dahlstrom, U., Fu, M. \& Lund, L. H. Atrial fibrillation in heart failure with preserved, mid-range, and reduced ejection fraction. JACC Heart Fail. $\mathbf{5}$ 565-574 (2017)

83. Lofman, I., Szummer, K., Dahlstrom, U., Jernberg, T. \& Lund, L. H. Associations with and prognostic impact of chronic kidney disease in heart failure with preserved, mid-range, and reduced ejection fraction. Eur. J. Heart Fail. 19, 1606-1614 (2017).

84. Lofman, I. et al. Incidence of, associations with and prognostic impact of worsening renal function in heart failure with different ejection fraction categories. Am. J. Cardiol. 124, 1575-1583 (2019).

85. Savarese, G. et al. Prevalence of, associations with, and prognostic role of anemia in heart failure across the ejection fraction spectrum. Int. J. Cardiol. 298, 59-65 (2020)

86. Streng, K. W. et al. Non-cardiac comorbidities in heart failure with reduced, mid-range and preserved ejection fraction. Int. J. Cardiol. 271, 132-139 (2018).

87. Solomon, S. D. et al. Influence of ejection fraction on outcomes and efficacy of spironolactone in patients with heart failure with preserved ejection fraction. Eur. Heart J. 37, 455-462 (2016).

88. Borlaug, B. A. et al. Effect of inorganic nitrite vs placebo on exercise capacity among patients with heart failure with preserved ejection fraction: the INDIE-HFpEF randomized clinical trial. JAMA 320, 1764-1773 (2018).
89. Redfield, M. M. et al. Isosorbide mononitrate in heart failure with preserved ejection fraction. N. Engl. J. Med. 373, 2314-2324 (2015).

90. Savji, N. et al. The association of obesity and cardiometabolic traits with incident $\mathrm{HFpEF}$ and HFrEF. JACC Heart Fail. 6, 701-709 (2018)

91. Pandey, A. et al. Relationship between physical activity, body mass index, and risk of heart failure. J. Am. Coll. Cardiol. 69, 1129-1142 (2017).

92. Pandey, A. et al. Physical activity, fitness, and obesity in heart failure with preserved ejection fraction. JACC Heart Fail. 6, 975-982 (2018).

93. Rao, V. N. et al. Adiposity and incident heart failure and its subtypes: MESA (Multi-Ethnic Study of Atherosclerosis). JACC Heart Fail. 6, 999-1007 (2018).

94. Bartko, P. E. et al. Secondary valve regurgitation in patients with heart failure with preserved ejection fraction, heart failure with mid-range ejection fraction and heart failure with reduced ejection fraction. Eur. Heart J. 41, 2799-2810 (2020).

95. Ghio, S. et al. Different correlates but similar prognostic implications for right ventricular dysfunction in heart failure patients with reduced or preserved ejection fraction. Eur. J. Heart Fail. 19 873-879 (2017).

96. Li, D. L., Quispe, R., Onyekwelu, C., Faillace, R. T $\&$ Taub, C. C. Racial differences of heart failure with midrange ejection fraction (HFmrEF): a large urban centre-based retrospective cohort study in the USA BMJ Open 9, e026479 (2019).

97. Jimenez-Marrero, S. et al. Sympathetic activation and outcomes in chronic heart failure: does the neurohormonal hypothesis apply to mid-range and preserved ejection fraction patients? Eur. J. Intern. Med. 81, 60-66 (2020).

98. Vergaro, G. et al. Sympathetic and renin-angiotensinaldosterone system activation in heart failure with preserved, mid-range and reduced ejection fraction. Int. J. Cardiol. 296, 91-97 (2019).

99. Cleland, J. G. F. et al. Beta-blockers for heart failure with reduced, mid-range, and preserved ejection fraction: an individual patient-level analysis of double-blind randomized trials. Eur. Heart J. 39, 26-35 (2018)

100. Lauritsen, J., Gustafsson, F. \& Abdulla, J. Characteristics and long-term prognosis of patients with heart failure and mid-range ejection fraction compared with reduced and preserved ejection fraction: a systematic review and meta-analysis. ESC Heart Fail. 5, 685-694 (2018).

101. Cheng, R. K. et al. Outcomes in patients with heart failure with preserved, borderline, and reduced ejection fraction in the Medicare population. Am. Heart J. 168, 721-730 (2014).

102. Pocock, S. J. et al. Predicting survival in heart failure: a risk score based on 39372 patients from 30 studies. Eur. Heart J. 34, 1404-1413 (2013).

103. Levy, W. C. et al. The Seattle Heart Failure Model: prediction of survival in heart failure. Circulation 113 , 1424-1433 (2006).

104. Simpson, J. et al. Prognostic models derived in PARADIGM-HF and validated in atmosphere and the swedish heart failure registry to predict mortality and morbidity in chronic heart failure. JAMA Cardiol. $\mathbf{5}$, 432-441 (2020)

105. Parikh, M. N., Lund, L. H., Goda, A. \& Mancini, D. Usefulness of peak exercise oxygen consumption and the heart failure survival score to predict survival in patients $>65$ years of age with heart failure. Am. J. Cardiol. 103, 998-1002 (2009).

106. Sartipy, U., Dahlstrom, U., Edner, M. \& Lund, L. H. Predicting survival in heart failure: validation of the MAGGIC heart failure risk score in 51,043 patients from the Swedish heart failure registry. Eur. J. Hear Fail. 16, 173-179 (2014).

107. Yang, S., Lund, L. H., Li, S., Dahlstrom, U., Sartipy, U. \& Levy, V. Validation of the Seattle heart failure model in the Swedish heart failure registry (abstr.). Circulation 140, A9844 (2019).

108. Goliasch, G. et al. Refining the prognostic impact of functional mitral regurgitation in chronic heart failure. Eur. Heart J. 39, 39-46 (2018).

109. Priori, S. G. et al. 2015 ESC Guidelines for the management of patients with ventricular arrhythmias and the prevention of sudden cardiac death: the Task Force for the Management of Patients with Ventricular Arrhythmias and the Prevention of Sudden Cardiac Death of the European Society of Cardiology (ESC). Endorsed by: Association for European Paediatric and Congenital Cardiology (AEPC). Eur. Heart J. 36 , 2793-2867 (2015). 
110. Vaduganathan, M. et al. Mode of death in heart failure with preserved ejection fraction. $J$. Am . Coll. Cardiol. 69, 556-569 (2017)

111. Bilchick, K. C., Stukenborg, G. J., Kamath, S. \& Cheng, A. Prediction of mortality in clinical practice for medicare patients undergoing defibrillator implantation for primary prevention of sudden cardiac death. J. Am. Coll. Cardiol. 60, 1647-1655 (2012).

112. Shadman, R. et al. A novel method to predict the proportional risk of sudden cardiac death in heart failure: derivation of the seattle proportional risk model. Heart Rhythm. 12, 2069-2077 (2015).

113. Bilchick, K. C. et al. Seattle heart failure and proportional risk models predict benefit from implantable cardioverter-defibrillators. J. Am. Coll. Cardiol. 69, 2606-2618 (2017)

114. Halliday, B. P. et al. Association between midwall late gadolinium enhancement and sudden cardiac death in patients with dilated cardiomyopathy and mild and moderate left ventricular systolic dysfunction. Circulation 135, 2106-2115 (2017).

115. Lam, C. S. \& Teng, T. H. Understanding heart failure with mid-range ejection fraction. JACC Heart Fail. 4 473-476 (2016)

116. Greenberg, B. Heart failure preserved ejection fraction with coronary artery disease: time for a new classification? J. Am. Coll. Cardiol. 63, 2828-2830 (2014).

117. Hwang, S. J., Melenovsky, V. \& Borlaug, B. A. Implications of coronary artery disease in heart failure with preserved ejection fraction. J. Am. Coll. Cardiol. 63, 2817-2827 (2014)

118. Lupon, J. et al. Heart failure with preserved ejection fraction infrequently evolves toward a reduced phenotype in long-term survivors. Circ. Heart Fail. 12, e005652 (2019).

119. Rusinaru, D. et al. Coronary artery disease and 10-year outcome after hospital admission for heart failure with preserved and with reduced ejection fraction. Eur. J. Heart Fail. 16, 967-976 (2014).

120. Savarese, G. et al. Reductions in $\mathrm{N}$-terminal pro-brain natriuretic peptide levels are associated with lower mortality and heart failure hospitalization rates in patients with heart failure with mid-range and preserved ejection fraction. Circ. Heart Fail. 9 , e003105 (2016)

121. Savarese, G. et al. Changes of natriuretic peptides predict hospital admissions in patients with chronic heart failure. A meta-analysis. JACC: Heart Fail. 2 148-158 (2014).

122. Savarese, G. et al. Prevalence and prognostic implications of longitudinal ejection fraction change in heart failure. JACC Heart Fail. 7, 306-317 (2019).

123. Kim, Y. J. \& Kim, R. J. The role of cardiac MR in new-onset heart failure. Curr. Cardiol. Rep. 13, 185-193 (2011).

124. Celutkiene, J. et al. Innovative imaging methods in heart failure: a shifting paradigm in cardiac assessment. Position statement on behalf of the Heart Failure Association of the European Society of Cardiology. Eur. J. Heart Fail. 20, 1615-1633 (2018).

125. Quarta, G. et al. Cardiac magnetic resonance in heart failure with preserved ejection fraction: myocyte, interstitium, microvascular, and metabolic abnormalities. Eur. J. Heart Fail. 22, 1065-1075 (2020).

126. Gulati, A. et al. Association of fibrosis with mortality and sudden cardiac death in patients with nonischemic dilated cardiomyopathy. JAMA 309, 896-908 (2013).

127. Klem, I. et al. Assessment of myocardial scarring improves risk stratification in patients evaluated for cardiac defibrillator implantation. J. Am. Coll. Cardiol. 60, 408-420 (2012).

128. Potter, E. \& Marwick, T. H. Assessment of left ventricular function by echocardiography: the case for routinely adding global longitudinal strain to ejection fraction. JACC Cardiovasc. Imaging 11, 260-274 (2018).

129. Venkateshvaran, A., Manouras, A., Kjellstrom, B. \& Lund, L. H. The additive value of echocardiographic pulmonary to left atrial global strain ratio in the diagnosis of pulmonary hypertension. Int. J. Cardiol. 292, 205-210 (2019).

130. Kraigher-Krainer, E. et al. Impaired systolic function by strain imaging in heart failure with preserved ejection fraction. J. Am. Coll. Cardiol. 63, 447-456 (2014).
131. Stokke, T. M. et al. Geometry as a confounder when assessing ventricular systolic function: comparison between ejection fraction and strain. J. Am. Coll. Cardiol. 70, 942-954 (2017).

132. Kalam, K., Otahal, P. \& Marwick, T. H. Prognostic implications of global LV dysfunction: a systematic review and meta-analysis of global longitudinal strain and ejection fraction. Heart 100, 1673-1680 (2014).

133. Stanton, T., Leano, R. \& Marwick, T. H. Prediction of all-cause mortality from global longitudinal speckle strain: comparison with ejection fraction and wall motion scoring. Circ. Cardiovasc. Imaging 2,356-364 (2009).

134. Adamo, L. et al. Proteomic signatures of heart failure in relation to left ventricular ejection fraction. J. Am. Coll. Cardiol. 76, 1982-1994 (2020).

135. Trivieri, M. G. et al. Challenges in cardiac and pulmonary sarcoidosis: JACC state-of-the-art review. J. Am. Coll. Cardiol. 76, 1878-1901 (2020).

136. Gigli, M. et al. Genetic risk of arrhythmic phenotypes in patients with dilated cardiomyopathy. J. Am. Coll. Cardiol. 74, 1480-1490 (2019).

137. Wahbi, K. et al. Development and validation of a new risk prediction score for life-threatening ventricular tachyarrhythmias in laminopathies. Circulation 140 293-302 (2019)

138. Wilcox, J. E., Fang, J. C., Margulies, K. B. \& Mann, D. L. Heart failure with recovered left ventricular ejection fraction: JACC scientific expert panel. J. Am. Coll. Cardiol. 76, 719-734 (2020).

139. Kramer, D. G. et al. Quantitative evaluation of drug or device effects on ventricular remodeling as predictors of therapeutic effects on mortality in patients with heart failure and reduced ejection fraction: a meta-analytic approach. J. Am. Coll. Cardiol. 56, 392-406 (2010).

140. Ueda, T. et al. Left ventricular ejection fraction (EF) of $55 \%$ as cutoff for late transition from heart failure (HF) with preserved EF to HF with mildly reduced $\mathrm{EF}$ Circ. J. 79, 2209-2215 (2015).

141. Januzzi, J. L. Jr. et al. Association of change in $\mathrm{N}$-terminal pro-B-type natriuretic peptide following initiation of sacubitril-valsartan treatment with cardiac structure and function in patients with heart failure with reduced ejection fraction. JAMA 322 1085-1095 (2019).

142. Desai, A. S. et al. Effect of sacubitril-valsartan vs enalapril on aortic stiffness in patients with heart failure and reduced ejection fraction: a randomized clinical trial. JAMA 322, 1077-1084 (2019).

143. Lupon, J. et al. Recovered heart failure with reduced ejection fraction and outcomes: a prospective study. Eur. J. Heart Fail. 19, 1615-1623 (2017).

144. Brann, A., Janvanishstaporn, S. \& Greenberg, B. Association of prior left ventricular ejection fraction with clinical outcomes in patients with heart failure with midrange ejection fraction. JAMA Cardiol. 5, 1027-1035 (2020).

145. Lupon, J. et al. Dynamic trajectories of left ventricular ejection fraction in heart failure. J. Am. Coll. Cardiol. 72, 591-601 (2018)

146. Cleland, J. G. et al. The perindopril in elderly people with chronic heart failure (PEP-CHF) study. Eur. Heart J. 27, 2338-2345 (2006)

147. Flather, M. D. et al. Randomized trial to determine the effect of nebivolol on mortality and cardiovascular hospital admission in elderly patients with heart failure (SENIORS). Eur. Heart J. 26, 215-225 (2005).

148. US National Library of Medicine. ClinicalTrials.gov https://clinicaltrials.gov/ct2/show/NCT03057951 (2021).

149. US National Library of Medicine. ClinicalTrials.gov https://clinicaltrials.gov/ct2/show/NCT03448406 (2020).

150. US National Library of Medicine. ClinicalTrials.gov https://clinicaltrials.gov/ct2/show/NCT03877224 (2020).

151. Lund, L. H., Benson, L., Dahlstrom, U., Edner, M. \& Friberg, L. Association between use of beta-blockers and outcomes in patients with heart failure and preserved ejection fraction. JAMA 312, 2008-2018 (2014).

152. Savarese, G. et al. Comorbidities and cause-specific outcomes in heart failure across the ejection fraction spectrum: A blueprint for clinical trial design Int. J. Cardiol. 313, 76-82 (2020)

153. Ibrahim, N. E. et al. Heart failure with mid-range ejection fraction: characterization of patients from the
PINNACLE registry(R). ESC. Heart Fail 6, 784-792 (2019).

154. Halliday, B. P. et al. Withdrawal of pharmacological treatment for heart failure in patients with recovered dilated cardiomyopathy (TRED-HF): an open-label, pilot, randomised trial. Lancet 393. 61-73 (2019).

155. Rogers, J. K. et al. Analysing recurrent hospitalizations in heart failure: a review of statistical methodology, with application to CHARM-Preserved. Eur. $J$. Heart Fail. 16, 33-40 (2014).

156. Alzahrani, T., Tiu, J., Panjrath, G \& Solomon, A. The effect of angiotensin-converting enzyme inhibitors on clinical outcomes in patients with ischemic cardiomyopathy and midrange ejection fraction: a post hoc subgroup analysis from the PEACE trial. Ther. Adv. Cardiovasc. Dis. 12, 351-359 (2018).

157. Girerd, N., Ferreira, J. P., Rossignol, P. \& Zannad, F. A tentative interpretation of the TOPCAT trial based on randomized evidence from the brain natriuretic peptide stratum analysis. Eur. J. Heart Fail. 18, 1411-1414 (2016).

158. FDA. Final Summary Minutes of the Cardiovascular and Renal Drugs Advisory Committee Meeting December 16, 2020. https://www.fda.gov/media/ 145548/download (2020).

159. Abdul-Rahim, A. H. et al. Effect of digoxin in patients with heart failure and mid-range (borderline) left ventricular ejection fraction. Eur. J. Heart Fail. 20, 1139-1145 (2018)

160. U.S. Food and Drug Administration. Highlights of Prescribing Information - Sacubitril/Valsartan https://www.accessdata.fda.gov/drugsatfda_docs/ label/2021/207620s018lbl.pdf (2020).

161. Seferovic, P. M. et al. Clinical practice update on heart failure 2019: pharmacotherapy, procedures, devices and patient management. An expert consensus meeting report of the Heart Failure Association of the European Society of Cardiology. Eur. J. Heart Fail. 21 1169-1186 (2019).

162. Savarese, G. et al. Non-insulin antihyperglycaemic drugs and heart failure: an overview of current evidence from randomized controlled trials. ESC Heart Fail. 7, 3438-3451 (2020).

163. Kini, V. et al. Appropriateness of primary prevention implantable cardioverter-defibrillators at the time of generator replacement: are indications still met? J. Am. Coll. Cardiol. 63, 2388-2394 (2014)

164. Adabag, S. et al. Association of implantable cardioverter defibrillators with survival in patients with and without improved ejection fraction: secondary analysis of the sudden cardiac death in heart failure trial. JAMA Cardiol. 2, 767-774 (2017)

165. No Authors Listed. Randomised, placebo-controlled trial of carvedilol in patients with congestive heart failure due to ischaemic Heart Disease. Australia/ New Zealand Heart Failure Research Collaborative Group. Lancet 349, 375-380 (1997)

166. Braunwald, E. et al. Angiotensin-converting-enzyme inhibition in stable coronary artery disease. N. Engl. J. Med. 351, 2058-2068 (2004).

167. Ahmed, A. et al. Effects of digoxin on morbidity and mortality in diastolic heart failure: the ancillary digitalis investigation group trial. Circulation 114 397-403 (2006).

168. Linde, C. et al. Cardiac resynchronization therapy in chronic heart failure with moderately reduced left ventricular ejection fraction: lessons from the Multicenter InSync Randomized Clinical Evaluation MIRACLE EF study. Int. J. Cardiol. 202, 349-355 (2016).

169. Yamamoto, K., Origasa, H., Hori, M. \& Investigators, J. D. Effects of carvedilol on heart failure with preserved ejection fraction: the Japanese Diastolic Heart Failure Study (J-DHF). Eur. J. Heart Fail. 15, 110-118 (2013).

170. US National Library of Medicine. ClinicalTrials gov https://clinicaltrials.gov/ct2/show/NCT03988634 (2021).

171. US National Library of Medicine. ClinicalTrials.gov https://clinicaltrials.gov/ct2/show/NCT04435626 (2021).

172. Pfeffer, M. A. et al. Regional variation in patients and outcomes in the treatment of preserved cardiac function heart failure with an aldosterone antagonist (TOPCAT) trial. Circulation 131, 34-42 (2015).

173. McGowan, J. H. \& Cleland, J. G. Reliability of reporting left ventricular systolic function by echocardiography: a systematic review of 3 methods. Am. Heart J. 146, 388-397 (2003). 
174. Sievers, B. et al. Visual estimation versus quantitative assessment of left ventricular ejection fraction: a comparison by cardiovascular magnetic resonance imaging. Am. Heart J. 150, 737-742 (2005).

175. Blondheim, D. S. et al. Reliability of visual assessment of global and segmental left ventricular function: a multicenter study by the Israeli Echocardiography Research Group. J. Am. Soc. Echocardiogr. 23, 258-264 (2010)

176. Lam, C. S. \& Solomon, S. D. Fussing over the middle child: heart failure with mid-range ejection fraction. Circulation 135, 1279-1280 (2017).

177. Pellikka, P. A. et al. Variability in ejection fraction measured by echocardiography, gated single-photon emission computed tomography, and cardiac magnetic resonance in patients with coronary artery disease and left ventricular dysfunction. JAMA Netw. Open 1, e181456 (2018).

\section{Acknowledgements}

L.H.L. is supported by the Karolinska Institutet, the Swedish Research Council (grant 523-2014-2336), the Swedish Heart Lung Foundation (grants 20150557, 20190310) and Stockholm County Council (grants 20170112, 20190525).

\section{Author contributions}

G. Savarese, D.S. and L.H.L. researched data for the article contributed substantially to discussion of the content and wrote the manuscript. All the authors reviewed and/or edited the manuscript before submission.

\section{Competing interests}

G. Savarese reports grants and personal fees from AstraZeneca and Vifor; grants and non-financial support from Boehringe Ingelheim; personal fees from Cytokinetics, GENESIS Medtronic, Radcliffe, Roche, Servier and Società Prodotti Antibiotici; and grants from Boston Scientific, Novartis and Pharmacosmos, unrelated to this Review. D.S. reports personal fees from GlaxoSmithKline, MSD and Novartis. L.H.L. reports personal fees from Abbott, Bayer, Lexicon, Medscape Merck, Myokardia, Pharmacosmos, Radcliffe Cardiology and Sanofi; grants and personal fees from AstraZeneca, Boehringer Ingelheim, Novartis and Vifor-Fresenius; and grants from Boston Scientific, unrelated to this Review. G. Sinagra declares no competing interests.

\section{Peer review information}

Nature Reviews Cardiology thanks B. A. Borlaug, W. J. Paulus and the other, anonymous, reviewer(s) for their contribution to the peer review of this work.

\section{Publisher's note}

Springer Nature remains neutral with regard to jurisdictional claims in published maps and institutional affiliations.

(c) Springer Nature Limited 2021 